\title{
Approximation of the Mean Escape Time for a Tilted Periodic Potential
}

\author{
Tamra Heberling ${ }^{1, *}$, Lisa Davis ${ }^{2}$ and Tomas Gedeon ${ }^{2}$ \\ ${ }^{1}$ Los Alamos National Laboratory, XTD-SS, Los Alamos, NM, 87545, USA. \\ 2 Department of Mathematical Sciences, Montana State University, Bozeman, MT, \\ 59717-2400, USA.
}

Communicated by Michel A. Van Hove

Received 12 June 2017; Accepted (in revised version) 13 December 2017

\begin{abstract}
We present a formula approximating the mean escape time (MST) of a particle from a tilted multi-periodic potential well. The potential function consists of a weighted sum of a finite number of component functions, each of which is periodic. For this particular case, the least period of the potential function is a common period amongst all of its component functions. An approximation of the MST for the potential function is derived, and this approximation takes the form of a product of the MSTs for each of the individual periodic component functions. Our first example illustrates the computational advantages of using the approximation for model validation and parameter tuning in the context of the biological application of DNA transcription. We also use this formula to approximate the MST for an arbitrary tilted periodic potential by the product of MSTs of a finite number of its Fourier modes. Two examples using truncated Fourier series are presented and analyzed.
\end{abstract}

AMS subject classifications: 60G10, 35Q84, 60J60, 60J65, 60H35

Key words: Brownian Ratchet, multi-periodic potential, tilted periodic potential, Fokker-Plank equation.

\section{Introduction}

Brownian ratchets were first introduced in the early 1900s by Smoluchowski and later developed by Feynman [23,51]. A Brownian ratchet is a system where periodic forcing coupled with Brownian motion can be harnessed and directed to do work, such as particle transport. Brownian ratchets are often modeled as particle transport through a periodic potential where the Brownian motion is incorporated as a thermal noise term

*Corresponding author. Email addresses: theberling@lanl.gov (T. Heberling), lisa.davis@montana.edu (L. Davis), tgedeon@montana.edu (T. Gedeon) 
used to propel the particle from one potential well, over a potential wall, and into the next well. Since the introduction of Brownian ratchets, they have been used in several fields to analyze diffusive motion, typically in microscopic systems where noise is fundamental to movement. Some examples include motor proteins [5], intercellular transport [6], DNA transcription [1,3,4,15,26, 27,32,49], Josephson junctions [12], ring-laser gyroscopes [13], and actin polymerization in neuroscience $[41,52]$.

Motivation for this work comes from seeking a model of RNA polymerase (RNAP) translocation along a DNA strand. RNAP motion is often modeled as a Brownian ratchet, where the periodic structure of $U(x)$ is aligned with RNAP transcription of successive nucleotides and the tilt $F x$ biases RNAP motion in one direction along the DNA strand. However, it is well-established in the literature that each RNAP experiences short pauses in its motion, where it stalls at some nucleotide location for a brief amount of time before resuming elongation. These so called transcriptional pauses occur at random locations with a mean frequency of about 1 per 100 nucleotides [39]. Therefore one particular model of RNAP motion on DNA with pauses may be a Brownian motion in tilted potential with $U(x)=U_{1}(x)+U_{2}(x)$, where $U_{1}(x, L)$ is periodic with period $L$ corresponding to 100 nucleotides and $U_{2}(x, L / 100)$ has a least period of $L / 100$.

We investigate a one-dimensional random walk generated by Gaussian white noise, $\xi(t)$, and influenced by a tilting force $F$. This stochastic model is formulated as

$$
\frac{d x}{d t}=-\frac{d}{d x}(U(x)-F x)+\sqrt{2 D} \xi(t), \quad t>0,
$$

where $x(t)$ is the one dimensional position at time $t$, and $D$ is the noise intensity. Often the parameter $D$ is given by the Einstein relation [18],

$$
D=\frac{k_{\beta} T}{\gamma}
$$

where $k_{\beta}$ is the Boltzmann constant, $T$ is temperature, and $\gamma$ is friction. The tilting force is included in the model by subtracting a tilting term, $F x$, from the periodic potential denoted as $U(x)$ in the equation above, see [43]. If $F<0$, the force pulls particles to the left and if $F>0$, the force pulls particles to the right. Using this tilting term, the effective potential in Eq. (1.1) is

$$
V(x)=U(x)-F x,
$$

where $U(x)$ is periodic with period $L$. One way to approach this problem is as Kramers problem in periodic potentials, to find the rate that a particle escapes from a potential well [31]. Kramers problem is an important area of research in this field $[8,9,11,38,46]$. Several exact solutions have been proposed and analyzed [20-22,36,37].

Our main motivation for interest in the mean escape time (MST) of a Brownian particle over a wall comes from the fact that the characteristics of the long range behavior of the particle (i.e. over many periods and a long time) can be computed by decomposing the motion into two components. One is the motion on the spatial scale of a single 
period, which is characterized by distribution of the escape time, and the other is on the spatial scale of many periods modeled as a random walk on segments. The time scale of the random walk is determined by the mean escape time from a single segment and therefore characterization of long term distribution of the particle in terms of drift and dispersion depends crucially on the MST. This decomposition is described in more detail in Section 2.

Characterization of the long range behavior of the particle depends explicitly on the form of the potential function that is specified. In this paper we study a tilted potential $V(x)$ whose periodic part $U(x)$, from Eq. (1.3), admits a decomposition into a sum of potentially simpler periodic functions $U_{i}(x)$

$$
U(x)=\sum_{i=1}^{n} U_{i}(x)
$$

where each $U_{i}(x)$ has a corresponding least period of

$$
L_{i}=\frac{L}{N_{i}}, \quad i=1,2, \cdots n \text { with integers } \quad 1=N_{1} \leq N_{2} \leq \cdots \leq N_{n} .
$$

Notice that all of the periodic functions, $U_{i}$, have a common period $L=L_{1}$. Our goal is to estimate the MST of a particle in the potential $V(x)$, given by Eq. (1.3), in terms of the MSTs of the individual components $U_{i}(x)$. Ideally such a formula would allow approximation of the MST for an arbitrary periodic potential by combining the MSTs of its Fourier modes. As we will see below, since computation of the MST of the potential $V(x)$ involves a numerical quadrature of a highly oscillatory function, by precomputing the MST of low frequency Fourier modes one is able to effectively approximate the MST of an arbitrary periodic function with efficiency.

We present several results in this direction. An issue to be addressed when decomposing $V(x)$ into its components, is how to assign the drift term $-F x$. We assign this term to the lowest frequency periodic function $U_{1}(x)$ and thus decompose the effective potential given by Eq. (1.3) into what we will refer to as the multi-periodic potential

$$
V^{n}(x)=\sum_{i=1}^{n} V_{i}(x)
$$

where

$$
V_{1}(x)=U_{1}(x)-F x \quad \text { and } \quad V_{i}(x)=U_{i}(x), \quad i=2, \cdots, n .
$$

We obtain an approximation formula for the MST corresponding to the potential in (1.3), and we derive an error estimate for the approximation. The error estimate for the approximate MST depends on the period of a low frequency function, rather than a high frequency function; as a result, our formula is most useful for periodic potentials with a dominant low frequency, perturbed by one or more high frequency functions. This error estimate also depends largely on the noise intensity $D$, with significantly less error 
computed when utilizing a large value of this parameter. The error estimate calculation and its dependency on parameters is discussed in detail in Section 4 . We also explore an equal distribution of the drift where we assign

$$
V_{i}^{n}(x)=U_{i}(x)-\frac{F}{n} x, \text { for } i=1, \cdots, n .
$$

We obtain the upper and lower bound on the MST of (1.3) for this case.

This paper is organized as follows. The main result is presented in Section 3 as a theorem with the proof of this theorem detailed in Sections 4 and 5. In Section 6 the MST approximation formula is combined with Fourier series expressions, and the examples of Section 7 illustrate both advantages and shortcomings of the approximation. In the next section, we give some preliminary but important concepts and formulas for diffusive transport in a tilted periodic potential.

\section{Transport in a tilted periodic potential}

We begin by formulating the Fokker-Planck equation for an ensemble of solutions of Eq. (1.1). We denote by $P\left(x, t \mid x_{0}, t_{0}\right)$ the probability that the particle is located at the spatial position $x$ at time $t$, given that the particle started at position $x_{0}$ at time $t_{0}$. We use the concise notation $P(x, t)$ when we fix $x_{0}=0$ and $t_{0}=0$. The time evolution of a statistical ensemble is captured by the time evolution of $P(x, t)$ which satisfies the Fokker-Planck equation $[10,33,42,46]$

$$
\partial_{t} P(x, t)=\partial_{x}\left(V^{\prime}(x)+D \partial_{x}\right) P(x, t) .
$$

While the Fokker-Planck equation is not solvable in general, the behavior of the solution can be qualitatively characterized by its drift and dispersion. Formulas for these quantities are given later in this section by Eq. (2.6) and Eq. (2.7) respectively. In our example of diffusive particles in a tilted potential, the mean of the function $P(x, t)$ will drift in the downhill direction of the tilt and its variance will grow (disperse) with time. We now review an elegant paper by Lindner et. al. [33] which derives explicit formulas for both the drift and dispersion.

The key idea of Lindner and co-authors is to separately solve a problem on two different spatial scales. First they partition the domain of the periodic potential into overlapping segments, $m_{i}$, of length $2 L$ (see Fig. 1) where $U(x)$ (defined in Eq. (1.3)) has least period $L$. In each segment, they solve the Fokker-Planck equation for the MST. In the second step, the MST is used to construct a stochastic jump process that transports the particle between the segments. We now describe the computation of the MST from a single segment. Consider a segment, $m_{i}$, on the spatial interval $x \in[-L, L]$ with initial data given by the Dirac delta function at $x=0$ that represents the initial position of the particle, and we impose absorbing boundary conditions at the endpoints, $x= \pm L$. When a particle reaches either $x= \pm L$ of segment $m_{i}$ it is removed from that segment and placed at the spatial location $x=0$ of the segment $m_{i+1}$ if it exits right, or the segment $m_{i-1}$ if the exit 


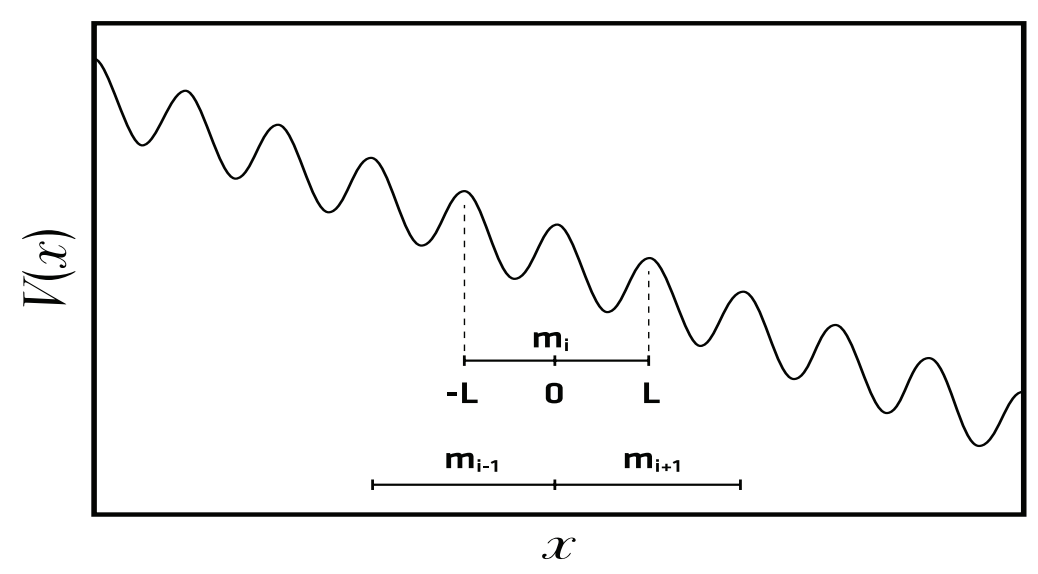

Figure 1: The domain of the potential function $V(x)$ is partitioned into overlapping segments of length $2 L$; here segment $m_{i}$ spans the interval $[-L, L]$. When a particle exits from either end of the segment, it is placed at the position $x=0$ of the neighboring segment.

is on the left, see Fig. 1. Since the escape from one segment is independent of all other segments, each segment can be solved by a Fokker-Planck equation in (2.1) with an initial condition and boundary conditions given by

$$
P(x, 0)=\delta(x), \quad P(-L, t)=P(L, t)=0 .
$$

Now we describe the larger spatial scale. Lindner et al. considers a stochastic jump process $n_{t}$ with jumps left and right depicted as realizations of $W_{i}=\{-1,1\}$ with probabilities $p_{-}$and $p_{+}$respectively. The net spatial movement at time $t$ is therefore a random sum of increments

$$
n_{t}=\sum_{i=1}^{N_{t}} W_{i}
$$

where $N_{t}$ is the total number of jumps before time $t$. Since each of the increments of $W_{i}$ as well as the escape times from a segment are independent of each other, $n_{t}$ is a Cumulative Process with Independent Increments [14,33]. Here the notation $N_{t}$ and $n_{t}$ is used to indicate a dependence on the time variable $t$, and it is not meant to denote a partial derivative with respect to $t$. The asymptotic mean and variance of $n_{t}$ are

$$
\left\langle n_{t}\right\rangle=\langle W\rangle \frac{t}{\mu}
$$

and

$$
\left\langle\Delta n_{t}^{2}\right\rangle=\left\langle\Delta W^{2}\right\rangle \frac{t}{\mu}+\langle W\rangle^{2} \frac{t \sigma^{2}}{\mu^{3}},
$$

where $\mu$ and $\sigma^{2}$ are the mean and variance of the escape time density from a single segment $m_{i}$, respectively [14]. The notation $\left\langle n_{t}\right\rangle$ represents the ensemble average of $n_{t}$, while 
$\left\langle\Delta n_{t}^{2}\right\rangle=\left\langle\left(n_{t}-\left\langle n_{t}\right\rangle\right)^{2}\right\rangle$ denotes the variance. Using these quantities, the drift, which for large times is given by the mean stationary velocity, is

$$
v=\frac{L\left(p_{+}-p_{-}\right)}{\mu}
$$

and the dispersion is given by the effective diffusion coefficient,

$$
D_{e f f}=\frac{L^{2}}{2}\left(\frac{1}{\mu}+\frac{\left(p_{+}-p_{-}\right)^{2}}{\mu^{3}}\left(\sigma^{2}-\mu^{2}\right)\right) .
$$

As one can observe from each of these equations, the MST from a single segment, denoted by $\mu$, is of central importance when describing diffusive transport of Brownian particles. More generally, the mean first passage time from a segment for a particle beginning at any position $x \in[-L, L]$ can be found using the techniques outlined in [24], as

$$
T_{1}(x)=\frac{1}{D}\left[\frac{\left(\int_{-L}^{x} \Phi(y) d y\right) \int_{x}^{L} \int_{-L}^{y} \frac{\Phi(y)}{\Phi(z)} d z d y-\left(\int_{x}^{L} \Phi(y) d y\right) \int_{-L}^{x} \int_{-L}^{y} \frac{\Phi(y)}{\Phi(z)} d z d y}{\int_{-L}^{L} \Phi(y) d y}\right]
$$

using the notation $\Phi(x)=e^{\frac{V(x)}{D}}$ for the sake of brevity. The MST, $\mu$, discussed here is simply $T_{1}(0)$, the first passage time when the particle begins at $x=0$. Hence we arrive at the equation which stands as the definition used in this work for the mean escape time of the particle from a single segment

$$
\mu=\frac{1}{D\left(1+e^{\frac{-F L}{D}}\right)} \int_{0}^{L} \int_{x-L}^{x} e^{\frac{V(x)}{D}} e^{\frac{-V(y)}{D}} d y d x:=q \iint_{\Omega} e^{\frac{V(x)}{D}} e^{\frac{-V(y)}{D}} d y d x .
$$

Here the parameter $q$ is defined as

$$
q:=\frac{1}{D\left(1+e^{\frac{-F L}{D}}\right)}
$$

and the domain $\Omega$ is defined as

$$
\Omega=\{(x, y) \mid x-L \leq y \leq x, 0 \leq x \leq L\} .
$$

For convenience we use $q$ and $\Omega$ to simplify the notation throughout this work.

\section{Mean escape time for a multi-periodic potential}

The main result of this paper concerns the MST of a Brownian particle from a segment of a tilted, periodic potential. The segments are fully explained in Section 2 and also 
depicted graphically in Fig. 1. The MST for a potential $V(x)$ of Eq. (1.3) is denoted by $\mu(V, L, F)$ and depends on three aspects of the potential: $V(x)$, the periodic function; $L$, the period; and $F$, the tilt. For simplicity, we will denote the MST as

$$
\mu:=\mu(V, L, F)
$$

calculated using Eq. (2.9).

Our main result approximates the MST for the multi-periodic potential defined by Eqs. (1.3), (1.4) and (1.5) in terms of the MSTs of the individual periodic functions $U_{i}(x)$. Since the potential $V(x)$ contains the tilting term $-F x$, this term has to be assigned to one of the component functions $U_{i}(x)$. In our first result, we assign the tilting term to the periodic component with the largest period $U_{1}(x)$, as given in Eq. (1.6), while the other periodic components will have zero tilt. We denote the MST for potential $V_{1}(x):=$ $U_{1}(x)-F x$, considered as a potential with period $L_{1}=L$ by

$$
\mu_{1}:=\mu\left(U_{1}, L_{1}, F\right) .
$$

When considering a potential function of the form given in Eq. (1.6) where the potential consists of a finite sum of periodic functions, we use the notation that for any integer $n$ the MST for the potential defined by $V^{n}$ is given by

$$
\mu_{n}=q \iint_{\Omega} e^{\frac{V^{n}(x)}{D}} e^{\frac{-V^{n}(y)}{D}} d y d x
$$

The following lemma provides a crucial scaling rule that allows us to define the appropriate notation for the MST approximation formula discussed in the next section.

Lemma 3.1. Consider the periodic potential $U(x) \in C[-L, L]$ with period $L$ and zero tilt. Then

$$
\mu(U, L, 0)=L^{2} \mu(\widehat{U}, 1,0),
$$

where $\widehat{U}(x)=U(L x)$ for all $x \in[-1,1]$.

Proof. First recall the definition

$$
\mu(U, L, 0)=\frac{1}{2 D} \int_{0}^{L} \int_{x-L}^{x} e^{\frac{U(x)}{D}} e^{\frac{-U(y)}{D}} d y d x
$$

Using the substitutions

$$
z=\frac{x}{L} \quad \text { and } \quad w=\frac{y}{L}
$$

the integral in Eq. (3.5) becomes

$$
\mu(U, L, 0)=\frac{1}{2 D} \int_{0}^{1} \int_{z-1}^{z} e^{\frac{\widehat{U}(z)}{D}} e^{-\frac{\widehat{U}(y)}{D}} L^{2} d y d z,
$$


where $\widehat{U}(z)=U(L z)$ for all $z \in[-1,1]$. Note here that $\widehat{U}$ is simply a transformed version of the original $U(x)$ function, and $\widehat{U}(z)$ is periodic with period 1. Applying the definition in Eq. (2.9) to the potential defined by $U(x)$ (with zero tilt, ie $F=0$ ), from Eq. (3.6), one can write

$$
\mu(U, L, 0)=(L)^{2} \mu(\widehat{U}, 1,0)
$$

where

$$
\mu(\widehat{U}, 1,0)=\frac{1}{2 D} \int_{0}^{1} \int_{z-1}^{z} e^{\frac{\widehat{U}(z)}{D}} e^{\frac{-\widehat{U}(y)}{D}} d y d z .
$$

The proof is completed.

The above equation gives the foundation for the definition below, and we use the notation

$$
v_{i}:=2 D \mu\left(\widehat{U}_{i}, 1,0\right), \quad \text { for } i=2,3, \cdots, n,
$$

where $\mu\left(\widehat{U}_{i}, 1,0\right)$ is the MST of the potential $\widehat{U}_{i}(z(x))$. Each function $\widehat{U}_{i}(z)$ is a transformed version of the original function $U_{i}(x)$ in a manner analogous to the process outlined in the proof above. For such a function, the potential $U_{i}(x)$, with period $L_{i}$, is defined on its domain of all $x \in\left[-L_{i}, L_{i}\right]$, and this interval is transformed using the linear mapping $z(x)=x / L_{i}$, where $z:\left[-L_{i}, L_{i}\right] \rightarrow[-1,1]$. The transformed function $\widehat{U}_{i}$ has period equal to one, and the notation $\widehat{U}_{i}(z, 1)$ is used to clarify this in the discussion that follows only when necessary. The constant $2 D$ in definition (3.9) simplifies the result in the definition and the theorem given below.

We now define an approximation of the MST for the function $V(x)$ using the notation

$$
T^{n}=\mu_{1}\left(\prod_{i=2}^{n} v_{i}\right),
$$

where $\mu_{1}$ is defined in (3.2) and $v_{i}$ is defined in (3.9) above. The theorem below gives an assessment of the error incurred with this approximation. The notation $T^{n}$ utilizes $n$ to denote the number of periodic functions in the decomposition of $V(x)$ in Eq. (1.6). This is an approximation of the true MST of $V(x)$ as defined in the Eq. (3.3). The simplifying approximation $T^{n}$ as well as the magnitude of the error from the original MST $\mu_{n}$ are the main focus of the remainder of this paper. We also illustrate the effectiveness of the approximation with three examples. First, we state the theorem that is the main result of the paper.

Theorem 3.1. Suppose the tilted multi-periodic potential $V(x)$ defined in Eq. (1.6) belongs to $C^{1}[-L, L]$. Then the multiplicative approximation, denoted $T^{n}$, for the MST of $V(x)$ is given by Eq. (3.10), and

$$
\left|T^{n}-\mu_{n}\right| \leq C \frac{\kappa^{\frac{1}{D}}}{D^{2}}
$$

where the constant $C$ depends explicitly on the fixed value of $L$, the functional form of the potential $V(x)$ as well as the noise intensity $D$. The constant $\kappa$ also depends upon the prescribed potential $V(x)$ and its first derivative. 
The proof for Theorem 3.1 is provided in Section 5. For completeness, we also investigate a different treatment for the tilt, this is explained below. In Theorem 3.1, we assign the tilt $-F x$ to the periodic function $U_{1}(x)$. Now we consider the case of splitting the tilt evenly among all the periodic functions.

Theorem 3.2. Suppose the tilted, multi-periodic potential is given by

$$
V(x)=\sum_{i=1}^{n} V_{i}^{n}(x)
$$

similar to the potential in Eq. (1.6) except $V_{i}^{n}(x):=U_{i}\left(x, L / N_{i}\right)-\frac{F x}{n}$, where the tilt is split into $n$ equal parts. Each periodic function has a tilting term associated with it. Then the MST, $\mu$, from a segment of this potential satisfies

$$
\begin{aligned}
& \frac{\left(1+e^{\frac{-F L}{n D}}\right)}{\left(1+e^{\frac{-F L}{D}}\right)}\left(\prod_{i=2}^{n} e^{\frac{F\left(1-N_{i}\right) L_{i}}{n D}} \eta_{i}\right) \mu\left(U_{1}, L_{1}, F / n\right)+\mathcal{O}\left(L_{2}\right) \\
& \leq \mu \leq \frac{\left(1+e^{\frac{-F L}{n D}}\right)}{\left(1+e^{\frac{-F L}{D}}\right)}\left(\prod_{i=2}^{n} \eta_{i}\right) \mu\left(U_{1}, L_{1}, F / n\right)+\mathcal{O}\left(L_{2}\right),
\end{aligned}
$$

where $\eta_{i}=D\left(1+e^{\frac{-F L_{i}}{n D}}\right) \mu\left(\widehat{U}_{i}, 1, \frac{F L_{i}}{n}\right)$.

The proof of Theorem 3.2 is provided in Appendix A. There is an important disadvantage to splitting the tilt term, aside from the fact that we are only able to provide lower and upper bounds. When Theorem 3.2 is used for a Fourier series approximation, increasing the number of Fourier terms approximating a periodic function $U(x)$ from $n$ to $n+1$ would be computationally expensive as all numbers $\eta_{i}$ would need to be recomputed. This is because the drift term in $V_{i}^{n}(x)$ is $F x / n$ while the drift term in $V_{i}^{n+1}(x)$ is $F x /(n+1)$ affecting the MST calculation for each term. In contrast, in the formula for $T^{n}$ in Theorem 3.1, the term $\mu_{1}$ and all terms $v_{i}, i=2, \cdots, n$ remain the same between successive approximations with $n$ and $n+1$ elements. Only the new term $v_{n+1}$ needs to be computed and inserted into formula (3.10) with $n+1$ elements. This is an advantage to using the MST given in Theorem 3.1.

\section{Preliminary results}

In this section we provide several lemmas that aid in the proof of Theorem 3.1. First we present a theorem from [24] that shows the probability of escaping the segment $m_{i}$ from Fig. 1 on the left or on the right is independent of the particular algebraic form of the periodic potential so long as the period $L$ and the tilt $F$ are fixed. We denote the probability of exiting to the right through the position $x=L$ by $p_{+}$, and the probability 
of escaping left through the position $x=-L$ by $p_{-}$. The probability $p_{-}$is related to the probability $p_{+}$through the equation

$$
p_{-}+p_{+}=1
$$

since a particle will leave the segment with probability one. Using formulas from [24], another relationship between these probabilities is also shown below.

Theorem 4.1. Let $U(x, L) \in C^{1}[-L, L]$ denote a periodic function with period L. Then $V(x)=$ $U(x, L)-F x$ is a tilted periodic potential function, and the escape probabilities associated with this potential function satisfy

$$
p_{+}=e^{\frac{F L}{D}} p_{-} .
$$

Corollary 4.1. Consider the potential function $V(x)=\sum_{i=1}^{n} U_{i}\left(x, L_{i}\right)-F x$ and the potential function $V_{1}(x)=U_{1}\left(x, L_{1}\right)-F x$. Since $L_{1}=L$, the common period of $V(x)$ and $V_{1}(x)$, then the probabilities of escaping to the right or to the left, respectively, are the same for both of the two potentials. Furthermore, these common values are

$$
p_{+}=\frac{1}{1+e^{\frac{-F L}{D}}}
$$

and

$$
p_{-}=\frac{e^{\frac{-F L}{D}}}{1+e^{\frac{-F L}{D}}} .
$$

The proof of Theorem 3.1 employs the Weighted Mean Value Theorem for Double Integrals. For the reader's convenience, the statement of this theorem is provided below.

Theorem 4.2 (Weighted Mean Value Theorem for Double Integrals). Let $f$ and $g$ be continuous functions on a closed and bounded domain $\Omega$. If $g$ is positive then there exists a point $\left(s^{\star}, t^{\star}\right) \in \Omega$ such that

$$
\iint_{\Omega} f(x, y) g(x, y) d y d x=f\left(s^{\star}, t^{\star}\right) \iint_{\Omega} g(x, y) d y d x .
$$

A proof of this theorem for single integrals is presented in [2], and the proof for the case of double integrals is presented in Appendix B.

Now we present a series of lemmas that provide estimates for the proof of Theorem 3.1. In the first lemma we estimate the integral for the MST given by Eq. (2.9). Note that the domain of integration is a parallelogram of length and height $L$, defined previously in (2.10). For a given integer $n$, we partition $\Omega$ into smaller parallelograms of length and height $L_{n}=L / N_{n}$, where $L_{n}$ is the period of $U_{n}(x)$ and the shortest of the periods $L_{i}, i=1, \cdots, n$. These smaller parallelograms are defined as

$$
\Omega_{k, s}:=\left\{(x, y) \mid(k-1) L_{n} \leq x \leq k L_{n}, x-L+(s-1) L_{n} \leq y \leq x-L+s L_{n}\right\}
$$


for each choice of $(k, s), k=1, \cdots, N_{n}, s=1, \cdots, N_{n}$, where $k$ partitions the domain on the $x$-axis, and $s$ partitions the domain on the $y$-axis. This is depicted in Fig. 2 . In the following results, we often use the reference domain or the canonical domain $\Omega_{0, n}$, which is the parallelogram that includes the origin, i.e.

$$
\Omega_{0, n}:=\left\{(x, y) \mid 0 \leq x \leq L_{n}, x-L_{n} \leq y \leq x\right\} .
$$

In the proof below we will also separate the function $U_{n}(x)$ from the multi-periodic potential (1.6) rewritten as

$$
V(x)=V^{n-1}(x)+U_{n}(x),
$$

where

$$
V^{n-1}(x)=\sum_{i=1}^{n-1} U_{i}(x)-F x
$$

Lemma 4.1. Consider a tilted multi-periodic potential $V(x) \in C^{1}[-L, L]$ given by Eq. (4.6). Then there exists a set of points $\left(x_{k, s}^{\star}, y_{k, s}^{\star}\right) \in \Omega_{k, s}$ for each $1 \leq k, s \leq N_{n}$ such that the MST for $V(x)$ is

$$
\mu=\frac{2 \mu\left(U_{n}, L_{n}, 0\right)}{\left(1+e^{\frac{-F L}{D}}\right)} \sum_{k=1}^{N_{n}} \sum_{s=1}^{N_{n}} \frac{\Psi_{n-1}\left(x_{k, s}^{\star}\right)}{\Psi_{n-1}\left(y_{k, s}^{\star}\right)},
$$

where the scalar valued function $\Psi_{n}$ is defined for any integer $n$ as

$$
\Psi_{n}(z):=e^{\frac{V^{n}(z)}{D}} .
$$

Proof. Using Eq. (2.9) we write the MST for $V(x)$ as

$$
\mu=q \iint_{\Omega} \frac{\Psi_{n-1}(x)}{\Psi_{n-1}(y)} e^{\frac{U_{n}(x)}{D}} e^{\frac{-U_{n}(y)}{D}} d y d x .
$$

Partitioning the domain $\Omega$ into subdomains $\Omega_{k, s}$ as described above (see Fig. 2), we have

$$
\mu=q \sum_{k=1}^{N_{n}} \sum_{s=1}^{N_{n}} \iint_{\Omega_{k, s}} \frac{\Psi_{n-1}(x)}{\Psi_{n-1}(y)} e^{\frac{U_{n}(x)}{D}} e^{\frac{-U_{n}(y)}{D}} d y d x .
$$

Using Theorem 4.2, for each pair $(k, s)$ there exists a point $\left(x_{k, s}^{\star} y_{k, s}^{\star}\right) \in \Omega_{k, s}$ such that

$$
\mu=q \sum_{k=1}^{N_{n}} \sum_{s=1}^{N_{n}} \frac{\Psi_{n-1}\left(x_{k, s}^{\star}\right)}{\Psi_{n-1}\left(y_{k, s}^{\star}\right)} \iint_{\Omega_{k, s}} e^{\frac{U_{n}(x)}{D}} e^{\frac{-U_{n}(y)}{D}} d y d x
$$

We next transform each domain $\Omega_{k, s}$ to the canonical domain $\Omega_{0, n}$ by an area-preserving, linear change of variables $G_{k, s}: \Omega_{k, s} \rightarrow \Omega_{0, n}$ defined by

$$
G_{k, s}(x, y)=\left(x-(k-1) L_{n}, y-\left(s-N_{n}\right) L_{n}-(k-1) L_{n}\right) .
$$




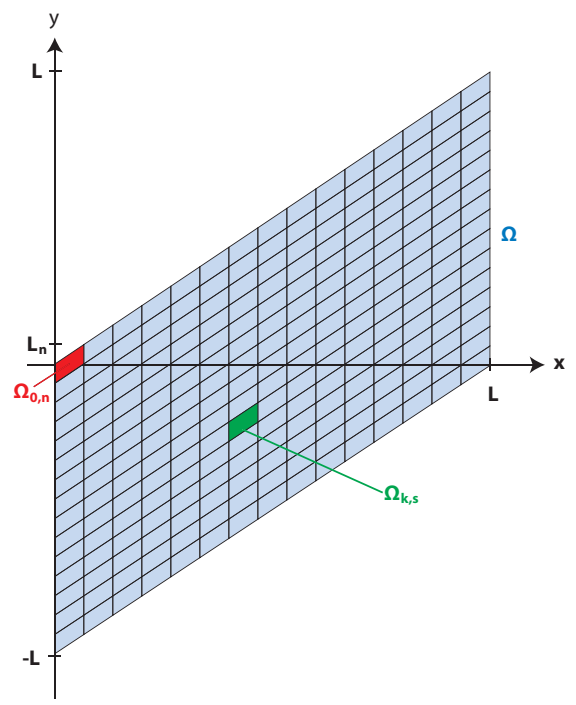

Figure 2: The domain of integration $\Omega$ is shown in blue, and it is partitioned into $N_{n}^{2}$ parallelograms, each with both width and height of $L_{n}$. These subdomains are denoted $\Omega_{k, s}$ for $1 \leq k, s \leq N_{n}$ with an arbitrary subdomain shown in green. The subdomain $\Omega_{0, n}$ is the parallelogram containing the origin shown in red.

Applying $G_{k, s}$ in order to map $\Omega_{k, s}$ to $\Omega_{0, n}$, exploiting the $L_{n}$-periodicity of the $U_{n}$ function within the integrand and multiplying and dividing by $2 D$ in (4.12), we have

$$
\mu=q(2 D) \sum_{k=1}^{N_{n}} \sum_{s=1}^{N_{n}} \frac{\Psi_{n-1}\left(x_{k, s}^{\star}\right)}{\Psi_{n-1}\left(y_{k, s}^{\star}\right)}\left(\frac{1}{2 D} \iint_{\Omega_{0, n}} e^{\frac{U_{n}(x)}{D}} e^{\frac{-U_{n}(y)}{D}} d y d x\right) .
$$

Note that the scaled integral term within the parentheses is the MST for the periodic potential $U_{n}(x)$ with zero tilt; that is, $\mu\left(U_{n}, L_{n}, 0\right)$. This term can be factored out of the summation. Hence Eq. (4.14) simplifies to Eq. (4.8), and the proof is complete.

The last preliminary result deals with estimation of the error between the double sum appearing in Eq. (4.8) and the double integral used in the MST calculations, as in Eq. (2.9). We begin by calculating the error over an arbitrary integration subdomain $\Omega_{k, s}$ and then sum the error estimates over all subdomains to compute the error over the entire domain $\Omega$. In particular, we define the difference between an appropriately scaled version of the double sum in (4.8) and the double integral for the MST of the multi-periodic potential $V(x)$ as

$$
\epsilon_{n}:=\left|\iint_{\Omega} \frac{\Psi(x)}{\Psi(y)} d y d x-\left(L_{n}\right)^{2} \sum_{k=1}^{N_{n}} \sum_{s=1}^{N_{n}} \frac{\Psi\left(x_{k, s}^{\star}\right)}{\Psi\left(y_{k, s}^{\star}\right)}\right|,
$$

where $\Psi(x)$ is defined as in Eq. (4.9) but we suppress the subscript notation for convenience. We also make the following definition of a constant needed for the proof of the 
result, and a bound is given that can easily be justified with elementary techniques. Let

$$
M_{2}=\max _{\left(z_{1}, z_{2}\right) \in \Omega}\left|\frac{\Psi\left(z_{1}\right)}{\Psi\left(z_{2}\right)}\right| \leq \kappa^{\frac{1}{D}},
$$

where $\mathcal{\kappa}$ is a constant depending on the choice of the periodic potential $V(x)$.

Lemma 4.2. Let $V \in C^{1}[-L, L]$, and consider a multi-periodic potential in the form (4.6). The error $\epsilon_{n}$ defined in (4.15) is bounded as follows

$$
\epsilon_{n}<C L_{n},
$$

where $C$ is a constant that depends on the functional form of $V$, the period $L$ and the noise intensity D.

Remark 4.1. The constant $C$ in Eq. (4.17) is independent of $n$, the number of periodic potentials used to form $V(x)$ in Eq. (1.6). Since each of the functions that form $V$ is uniformly bounded by a constant that is independent of $n$, the constant $C$ only depends on this bound, but not on $n$.

Proof. Consider the error $\epsilon_{k, s}$, over one arbitrary parallelogram $\Omega_{k, s}$. For simplicity the point $\left(x_{k, s}^{\star}, y_{k, s}^{\star}\right)$ is denoted as $\left(x^{\star}, y^{\star}\right)$. The error for each $\Omega_{k, s}$ is

$$
\epsilon_{k, s}=\left|\iint_{\Omega_{k, s}} \frac{\Psi(x)}{\Psi(y)} d y d x-\frac{\Psi\left(x^{\star}\right)}{\Psi\left(y^{\star}\right)}\left(L_{n}\right)^{2}\right| .
$$

Define the function $f(x, y)=\Psi(x) / \Psi(y)$, and for any $(x, y) \in \Omega_{k, s}$, the first-order Taylor expansion of the integrand, $f(x, y)$, in a neighborhood of $\left(x^{\star}, y^{\star}\right)$ is

$$
f(x, y)=\frac{\Psi\left(x^{\star}\right)}{\Psi\left(y^{\star}\right)}+\frac{V^{\prime}(a)}{D} \frac{\Psi(a)}{\Psi(b)}\left(x-x^{\star}\right)-\frac{V^{\prime}(b)}{D} \frac{\Psi(a)}{\Psi(b)}\left(y-y^{\star}\right),
$$

where $(a, b)$ is a point in $\Omega_{k, s}$ in a neighborhood of $\left(x^{\star}, y^{\star}\right)$ that depends on the point $(x, y)$. Then Eq. (4.18) is expressed as a single integral, and using the first order Taylor expansion in the integral gives

$$
\begin{aligned}
\epsilon_{k, s} & =\left|\iint_{\Omega_{k, s}} f(x, y)-f\left(x^{\star}, y^{\star}\right) d y d x\right| \\
& =\left|\iint_{\Omega_{k, s}} \frac{\Psi\left(x^{\star}\right)}{\Psi\left(y^{\star}\right)}+\frac{\Psi(a)}{\Psi(b)}\left[\frac{V^{\prime}(a)}{D}\left(x-x^{\star}\right)-\frac{V^{\prime}(b)}{D}\left(y-y^{\star}\right)\right]-\frac{\Psi\left(x^{\star}\right)}{\Psi\left(y^{\star}\right)} d y d x\right| .
\end{aligned}
$$

Since $V(x) \in C^{1}$, then $V(x)$ and its derivative is bounded on $[-L, L]$. We also replace $\left|x-x^{\star}\right|$ by the upper bound $L_{n}$, and $\left|y-y^{\star}\right|$ by the upper bound $2 L_{n}$. Using these and integrating over $\Omega_{k, s}$ gives the error estimate

$$
\begin{aligned}
\epsilon_{k, s} & \leq \frac{1}{D} \max _{z \in[-L, L]}\left|V^{\prime}(z)\right| M_{2}\left(\iint_{\Omega_{k, s}}\left|x-x^{\star}\right|+\left|y-y^{\star}\right| d y d x\right) \\
& \leq \frac{\kappa^{\frac{1}{D}}\left\|V^{\prime}\right\|_{\infty}}{D}\left(3 L_{n}^{3}\right) .
\end{aligned}
$$


Relabeling the constant in Eq. (4.21), we arrive at the error estimate over the subdomain $\Omega_{k, s}$

$$
\epsilon_{k, s} \leq \frac{\widehat{C}}{D}\left(L_{n}\right)^{3}, \quad \text { for } 1 \leq k, s \leq N_{n}
$$

and we note that the constant $\widehat{C}$ depends on both the functional form of $V$ (and its derivative) and the parameter $D$. Summing the error over the $N_{n}^{2}$ parallelograms we determine

$$
\epsilon_{n} \leq \frac{\widehat{C}}{D}\left(L_{n}\right)^{3} N_{n}^{2}=\frac{\widehat{C}}{D}(L)^{2} L_{n}=C L_{n}
$$

where $C=L^{2} \frac{\widehat{C}}{D}$ is a constant that depends explicitly on the fixed value of $L$, the functional form of the potential $V(x)$ as well as the noise intensity $D$.

\section{Proof of Theorem 3.1}

The proof of the main result of this paper is by recursion, and the following lemma is given in order to simplify the recursion step of the proof. In addition, the following definition generalizes the bound on $V^{\prime}(\cdot)$ used in Eq. (4.21) to include notation suitable for the recursion step used in the arguments given below. For a fixed positive integer $K$, define the bound

$$
M_{1, K}=\max _{z \in[-L, L]}\left|\frac{d}{d x} V^{K-1}(z)\right|=\left\|\frac{d}{d x} V^{K-1}\right\|_{\infty} .
$$

Lemma 5.1. Fix an integer $K \geq 1$, and assume that the potential $V^{K+1}$ is defined by (1.6) and its MST is given as in Eq. (3.3). If $\mu_{K}$ is known, then $\mu_{K+1}$ is given by

$$
\mu_{K+1}=v_{K+1}\left(\mu_{K}\right)+q v_{K+1} \epsilon_{K+1},
$$

where

$$
\left|\epsilon_{K+1}\right| \leq C_{K+1} L_{K+1} .
$$

Proof. Beginning with the definition in (3.3) and partitioning $\Omega$ into sub-parallelograms of size determined by $N_{K+1}$, we have

$$
\begin{aligned}
\mu_{K+1} & =q\left(\iint_{\Omega} e^{\frac{V^{K}(x)}{D}} e^{\frac{-V^{K}(y)}{D}} e^{\frac{u_{K+1}(x)}{D}} e^{\frac{-U_{K+1}(y)}{D}} d y d x\right) \\
& =q \sum_{k=1}^{N_{K+1}} \sum_{s=1}^{N_{K+1}} \iint_{\Omega_{k, s}} \frac{\Psi_{K}(x)}{\Psi_{K}(y)} e^{\frac{U_{K+1}(x)}{D}} e^{\frac{-U_{K+1}(y)}{D}} d y d x,
\end{aligned}
$$

where $\Psi_{K}$ is given by (4.9), but in the discussion that follows, we suppress the subscript in order to simplify the notation. By Theorem 4.2 there exists a point $\left(x_{k, s}^{\star}, y_{k, s}^{\star}\right) \in \Omega_{k, s}$ for each $1 \leq k, s \leq N_{K+1}$ such that

$$
\mu_{K+1}=q \sum_{k=1}^{N_{K+1}} \sum_{s=1}^{N_{K+1}}\left[\frac{\Psi\left(x^{\star}\right)}{\Psi\left(y^{\star}\right)}\right] \iint_{\Omega_{k, s}} e^{\frac{u_{K+1}(x)}{D}} e^{\frac{-u_{K+1}(y)}{D}} d y d x,
$$


and note that the $k, s$ dependence of $x^{\star}$ and $y^{\star}$ is suppressed for simplicity.

Then the expression for $\mu_{K+1}$ can be rewritten by replacing the integral over $\Omega_{k, s}$ with the integral over the reference domain defined in Eq. (4.5) using the mapping in (4.13). The integral over $\Omega_{0, K+1}$ is independent of the summation, can be factored out and then is expressed in terms of the definition of $v_{K+1}$ as shown in the equations below.

$$
\begin{aligned}
\mu_{K+1} & =q \sum_{k=1}^{N_{K+1} N_{K+1}}\left[\frac{\Psi\left(x^{\star}\right)}{\Psi\left(y^{\star}\right)}\right] \iint_{\Omega_{0, K+1}} e^{\frac{u_{K+1}(x)}{D}} e^{\frac{-u_{K+1}(y)}{D}} d y d x \\
& =q(2 D) \mu\left(U^{K+1}, L_{K+1}, 0\right) \sum_{k=1}^{N_{K+1}} \sum_{s=1}^{N_{K+1}}\left[\frac{\Psi\left(x^{\star}\right)}{\Psi\left(y^{\star}\right)}\right] \\
& =\frac{q(2 D) \mu\left(U^{K+1}, L_{K+1}, 0\right)}{L_{K+1}^{2}} \sum_{k=1}^{N_{K+1}} \sum_{s=1}^{N_{K+1}}\left[\frac{\Psi\left(x^{\star}\right)}{\Psi\left(y^{\star}\right)}\right] L_{K+1}^{2} \\
& =q(2 D) \mu\left(\widehat{U}^{K+1}, 1,0\right) \sum_{k=1}^{N_{K+1}} \sum_{s=1}^{N_{K+1}}\left[\frac{\Psi\left(x^{\star}\right)}{\Psi\left(y^{\star}\right)}\right] L_{K+1}^{2} \\
& =q v_{K+1} \sum_{k=1}^{N_{K+1}} \sum_{s=1}^{N_{K+1}}\left(\iint_{\Omega_{k, s}}\left[\frac{\Psi(x)}{\Psi(y)} d y d x\right]+\epsilon_{k, s}\right) .
\end{aligned}
$$

The last equalities follow from applying Lemma 3.1 and using the definition of $v_{K+1}$ in Eq. (3.9) to simplify the term left of the summation, and the term within the parentheses is obtained using the result in Lemma 4.2. Applying the summation over all the parallelograms, the first term can then be written in terms of $\mu_{K}$, and the error terms can be expressed as follows

$$
\begin{aligned}
\mu_{K+1} & =v_{K+1}\left(q \iint_{\Omega} \frac{\Psi(x)}{\Psi(y)} d y d x+q \sum_{k=1}^{N_{K+1}} \sum_{s=1}^{N_{K+1}} \epsilon_{k, s}\right) \\
& =v_{K+1}\left(\mu_{K}\right)+q v_{K+1} \epsilon_{K+1},
\end{aligned}
$$

where $\epsilon_{k, s}$ and $\epsilon_{K+1}$ are exactly those given in Eqs. (4.22)-(4.23), and

$$
\left|\epsilon_{K+1}\right| \leq \frac{3\left\|\frac{d}{d x} V^{K}\right\|_{\infty} M_{2} L_{K+1}}{D} \leq \frac{3\left\|\frac{d}{d x} V^{K}\right\|_{\infty} K^{\frac{1}{D}}}{D} L_{K+1} \leq C_{K+1} L_{K+1} .
$$

The proof is completed.

With the three previous results, we are ready to prove Theorem 3.1.

Proof. Fix an integer $K \geq 1$, and the proof is constructed by applying Lemma 5.1 recursively to obtain

$$
\begin{aligned}
\mu_{K+1} & =v_{K+1}\left(\mu_{K}\right)+q v_{K+1} \epsilon_{K+1} \\
& =v_{K+1}\left(v_{K} \mu_{K-1}+q v_{K} \epsilon_{K}\right)+q v_{K+1} \epsilon_{K+1} \\
& =v_{K+1} v_{K}\left(v_{K-1} \mu_{K-2}+q\left(v_{K-1} \epsilon_{K-1}\right)\right)+q\left(v_{K} v_{K+1} \epsilon_{K}\right)+q v_{K+1} \epsilon_{K+1}
\end{aligned}
$$




$$
\begin{aligned}
& =\left(\prod_{i=K-1}^{K+1} v_{i}\right) \mu_{K-2}+q \sum_{i=K-1}^{K+1}\left(\prod_{j=i}^{K+1} v_{j}\right) \epsilon_{i} \\
& =\vdots \\
& =\left(\prod_{i=2}^{K+1} v_{i}\right) \mu_{1}+q \sum_{i=2}^{K+1}\left(\prod_{j=i}^{K+1} v_{j}\right) \epsilon_{i} \\
& =T^{K+1}+E(K+1),
\end{aligned}
$$

where the error term in the above equation is represented by $E(K+1)$. Then define

$$
B_{i}=\prod_{j=i}^{K+1} v_{j} \text { and } B=\max _{1 \leq i \leq K+1}\left\{B_{i}\right\},
$$

and we see that the error is bounded by the following.

$$
\begin{aligned}
|E(K+1)|=\left|\mu_{K+1}-T^{K+1}\right| & \leq q\left|\sum_{i=2}^{K+1}\left(\prod_{j=i}^{K+1} v_{j}\right) \epsilon_{i}\right| \\
& \leq q B \sum_{i=2}^{K+1}\left|\epsilon_{i}\right| \\
& \leq q B\left[\frac{3 M_{2}}{D} \sum_{i=2}^{K+1} M_{1, i} L_{i}\right] \\
& \leq \frac{q B \kappa^{\frac{1}{D}}}{D}\left[3 \sum_{i=2}^{K+1} M_{1, i} L_{i}\right] \\
& \leq C \frac{\kappa^{\frac{1}{D}}}{D^{2}} .
\end{aligned}
$$

Then the proof of Theorem 3.1 is completed.

The approximation $T^{N}$ of the MST $\mu_{N}$ gives reasonable accuracy and is computationally efficient for the repetitive trials of model validation. This is demonstrated in Section 7.1 where a model of RNAP transcription is tuned to satisfy certain biological characteristics. As seen in the following sections, $T^{N}$ is a reliable approximation of the MST, but it is not asymptotically convergent in the classical sense. When a Fourier series is used to construct a periodic potential function, the MST approximation, $T^{N}$, for the truncated series does not necessarily improve as we increase $N$, where $N$ is the number of component functions used to construct the truncated series. That is, $T^{N}$ does not generally converge to $\mu^{N}$ as $N \rightarrow \infty$. The error estimate in Eq. (5.9) shows that this can happen when the value of $B$ increases with the integer $K$ or when the value of $M_{1, K}$ increases with $K$.

Having a specific bound for the error is illuminating for several reasons. First, this error bound explicitly shows the dependence on the noise intensity $D$. The parameter $\kappa$ 
has $1 / D$ as an exponent and the $E(K+1)$ has $D^{2}$ in the denominator. This implies that the error bound will decrease with increasing $D$, but it will increase with decreasing $D$. Also, the constant $C$ contains the length parameter $L_{i}$. Since $L_{i}$ decreases with increasing $i$, the largest term of the error bound corresponds to the first term, $i=2$. Therefore, the approximation $T^{N}$ will be the most accurate in the case where $L_{2}<<L_{1}$. This corresponds to a case where there is a distinct separation of spatial scales over which each potential has the most influence on the MST. The example in Section 7.1 illustrates an application where this relationship can be exploited. There the potential function describing a transcriptional pause influences the MST calculation on a much smaller spatial scale than that of the potential describing the RNAP elongation mechanism.

The following section also ties this concept to that of the classical Fourier series approximation of a function and relates the MST of a truncated Fourier series to that of the full series. That is, it demonstrates that $\mu_{N} \rightarrow \mu$ as $N \rightarrow \infty$ under certain conditions, and the examples in Section 7 show that $T^{N}$ can be a very good approximation of $\mu_{N}$ even when convergence as $N \rightarrow \infty$ is not guaranteed.

\section{Fourier series expansion of a periodic potential}

When using a periodic potential, it is common to approximate the potential with a truncated Fourier series approximation. Typically one can achieve a good approximation to the original potential assuming a sufficient number of terms are present in the truncated series. For a function $U(x)$ that is periodic with period $L$ on the interval $[0, L]$, the Fourier series representation of $U(x)$ is

$$
U(x)=\frac{a_{0}}{2}+\sum_{n=1}^{\infty} a_{n} \cos \left(\frac{2 \pi n x}{L}\right)+\sum_{n=1}^{\infty} b_{n} \sin \left(\frac{2 \pi n x}{L}\right)
$$

and the truncated Fourier expansion is then

$$
U^{N}(x)=\frac{a_{0}}{2}+\sum_{n=1}^{N} a_{n} \cos \left(\frac{2 \pi n x}{L}\right)+\sum_{n=1}^{N} b_{n} \sin \left(\frac{2 \pi n x}{L}\right) .
$$

Following the Fourier Convergence Theorem, discussed in detail in [7] for example, if $U(x)$ and its derivative $U^{\prime}(x)$ are piecewise continuous, its Fourier series converges pointwise to $U(x)$ for all values $x$ where $U(x)$ is continuous. At points of discontinuity, $x=x_{0}$, the Fourier series converges to the average of the left and right limits of $U(x)$ as $x \rightarrow x_{0}$. The convergence of the Fourier series becomes uniform if we require that $U(x)$ is continuous with $U^{\prime}(x)$ piecewise continuous [7]. Using this result, a theorem for the convergence of $\mu_{N}$ to $\mu$ is presented below.

Theorem 6.1. Let $U(x)$ be a continuous periodic function with a piecewise continuous derivative, with $x \in[-L, L]$. If $U^{N}(x)$ represents the truncated Fourier series approximation of $U(x)$ given 
in Eq. (6.2) then the MST for $U^{N}(x)$ defined in (3.3) converges to the MST of $U(x)$; that is,

$$
\lim _{N \rightarrow \infty} \mu_{N}=\mu .
$$

Proof. Consider the periodic potential $U(x)-F x$ and the truncated Fourier series potential with $N$ terms $U^{N}(x)-F x$. Since $U(x)$ is a continuous function with a piecewise continuous derivative, by the Fourier Convergence Theorem $U^{N}(x)$ converges uniformly to $U(x)$ [7]. The uniform convergence implies that

$$
\iint_{\Omega} e^{\frac{U(x)-F x}{D}} e^{\frac{-(U(y)-F y)}{D}} d y d x=\lim _{N \rightarrow \infty} \iint_{\Omega} e^{\frac{U^{N}(x)-F x}{D}} e^{\frac{-\left(U^{N}(y)-F y\right)}{D}} d y d x
$$

(this follows directly from Theorem 7.16 in [47]). Therefore

$$
\begin{aligned}
\mu & =\frac{1}{D\left(1+e^{\frac{-F L}{D}}\right)} \iint_{\Omega} e^{\frac{U(x)-F x}{D}} e^{\frac{-(U(y)-F y)}{D}} d y d x \\
& =\lim _{N \rightarrow \infty} \frac{1}{D\left(1+e^{\frac{-F L}{D}}\right)} \iint_{\Omega} e^{\frac{U^{N}(x)-F x}{D}} e^{\frac{-\left(U^{N}(y)-F y\right)}{D}} d y d x=\lim _{N \rightarrow \infty} \mu_{N} .
\end{aligned}
$$

The proof is completed.

With the preceding result, ideally one would like to quantify the error of the MST of the truncated series based on the number of terms, $N$. That is, we would like to characterize the magnitude

$$
\left|\mu-\mu_{N}\right| \leq q \iint_{\Omega} e^{\frac{-F(x-y)}{D}}\left|e^{\frac{U(x)-U^{N}(x)}{D}}-e^{\frac{U(y)-U^{N}(y)}{D}}\right| d \Omega
$$

as a function of the number of terms included in the truncated series approximation. While this may be done for the error given by the $L^{2}$ norm [25], $\left\|U-U^{N}\right\|_{2}$, we are not able to do so using the error given by the pointwise difference $\left|U-U^{N}\right|$. In fact, Lemma 10.2 from [30] shows that the pointwise convergence of a Fourier series may be arbitrarily slow.

\section{Examples}

This section presents examples that illustrate various aspects of the theoretical results given in the paper. We explore three examples using several forms of MST calculations. For this purpose, the MST of the original potential given by Eq. (2.9) and that of the truncated Fourier series potential, are denoted as $\mu$ and $\mu_{N}$, respectively. That is, $\mu_{N}=\mu\left(U^{N}(x), 2 \pi, 0\right)$ as described in the notation of Eq. (3.1) where $U^{N}$ is defined in Eq. (6.2). Moreover, in the first example, a Fourier series is not used as the potential consists of a sum of two periodic potentials, and the notation $\mu_{2}$ represents the true MST 
for that situation. In the third case, we compute the multiplicative approximation of the MST given by Eq. (3.10) using a potential consisting of a finite sum of periodic functions (such as a truncated Fourier series potential when one is under consideration), and this is denoted $T^{N}$ to remain consistent with previous sections.

Section 7.1 illustrates an application of this research to a biological model of DNA transcription where several RNA polymerase are transcribing the DNA strand simultaneously. Their elongation is interrupted by short, frequent transcriptional pauses. The elongation process as well as the transcriptional pause process are each modeled as a periodic process using a periodic potential function. The period of the elongation process is determined by the average duration of the transcription of one nucleotide, and the period of the pause process is much longer. Pauses are observed to occur, on average, every 100 nucleotides [39] during RNAP elongation. We apply the MST approximation in Eq. (3.10) and explore the influence of the model parameters on the calculated MST.

The next example in Section 7.2 considers a quadratic wave as the periodic potential. We derive the Fourier series on the segment $[0, L]$ and compute the MST of the truncated series of the periodic function $U(x)$ in the potential $V(x)=U(x)-F x$. Of particular interest is the calculation of the multiplicative MST for the truncated Fourier expansion using Eq. (3.10) and measuring the errors when applying the approximation $T^{N}$ described in this work.

Employing Eq. (3.10) can be particularly advantageous where there is a large gap in Fourier series frequencies as is the special case for a periodic function $U_{1}(x, L)+$ $U_{2}(x, L / N)$ where $N$ is a large integer. Section 7.3 considers a function of this type where the multi-periodic potential is highly oscillatory over the interval $[0, L]$, which can lead to a numerical integration for the MST in Eq. (2.9) that is slow and often inefficient. With $N$ large, the largest term in the error for Eq. (3.10) is proportional to $L_{N}=\left(\frac{L}{N}\right)$, since the expression in the last steps of Eq. (5.9) simplifies to a nice form. Specifically, the term in the final summation becomes simply $3 M_{1, N} L_{N}$. In this case $L_{N}$ is small, and the numerical integration for the MST of the potential $V_{1}(x)=U_{1}(x)-F x$ and the potential $V_{2}(x)=U_{2}(x)$ are individually computed more easily and more efficiently by separate numerical integration schemes. In addition, those values can be computed offline and stored for later use if additional terms of the Fourier series are needed at a later date. The example in Section 7.3 illustrates the use of the approximation in this context, and the magnitude of the error as a function of the parameter $D$ is also explored.

\subsection{RNAP elongation in the presence of transcriptional pausing}

The original motivation for investigating the MST of periodic potentials was in the application of Brownian ratchets to the mathematical modeling of DNA transcription. The example given in this section illustrates how the MST approximation presented in the previous sections can be used for such an application. We model the transcription process using two periodic potentials, one for RNAP elongation and the other for RNAP pausing. These two components of the potential function contribute to the overall MST 
on separate spatial scales. The MST approximation derived in this work is advantageous for the biological application because we are able to vary parameters of the two periodic potentials independently and efficiently approximate relevant measures of model validity. Furthermore, modifications to the tilt parameter or other biologically relevant parameters only require changes in one of the two potentials, and we take advantage of this in our computations as well. The efficiency of calculation, combined with a small observed error, makes the approximation a useful tool to study parameter dependencies in this problem.

Elongation of RNAP along the DNA strand is not uniform but is interrupted by frequent transcriptional pauses. There are at least three different types of pauses; backtracking pauses, hairpin pauses, and ubiquitous pauses [17, 39]. Backtracking pauses and hairpin pauses have been shown to have a higher probability of occurring during transcription of specific sequences $[16,28,29]$. On the other hand, ubiquitous pauses are thought to have no dependence on DNA sequence and are equally likely to occur at any position along the DNA strand. It has been theorized that ubiquitous pauses are caused by a restructuring of the polymerase [39], but the exact cause remains an open question. These pauses are short (1-5 seconds) and occur approximately every 100 nucleotides (nt) [39].

The potential function for this example is specified by $V(x)=U_{1}\left(x, L_{1}\right)+U_{2}\left(x, L_{2}\right)-F x$ where $L_{1}=100 \mathrm{nt}$ and $L_{2}=1 \mathrm{nt}$. We assume that the periodic potentials have the form

$$
U_{1}(x)=c_{1} e^{\epsilon\left(\cos \left(\frac{2 \pi x}{L_{1}}\right)-1\right)}
$$

for the pause process experienced by the RNAP and

$$
U_{2}(x)=c_{2} \cos \left(\frac{2 \pi x}{L_{2}}\right)
$$

for the elongation motion of the RNAP, see Fig. 3. The higher amplitude spikes (such as the one in the middle of Fig. 3) correspond to positions where we expect the RNAP to pause as it will require more energy and therefore time, for a particle to exit a well with a larger amplitude. The smaller amplitude potential models the standard elongation that takes place between each pause. In the following section, the model parameters are quantified and used to tune the model to accurately depict RNAP movement.

\subsubsection{Estimation of parameter values.}

There are seven parameters in the transcription model; $D, c_{1}, c_{2}, L_{1}, L_{2}, F$, and $\epsilon$. The parameters $L_{1}=100$ and $L_{2}=1$ are mentioned above and defined as such because of the average number of nucleotides on the $r r n$ operon between RNAP pauses. Section 7.1.2 describes how the amplitude parameters $c_{1}$ and $c_{2}$ are calculated to reach the desired MST based on information reported in the biological literature. The noise intensity parameter, $D$, is computed from literature as well, and the tilt $F$ is computed as a function of $D$ as explained below. The only parameter that is not computed in a manner that is consistent 


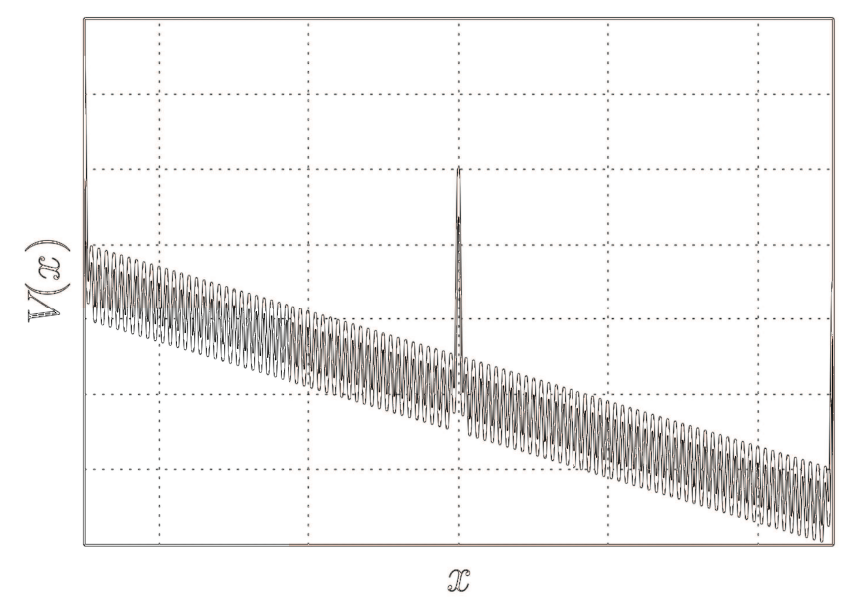

Figure 3: Plot of the RNAP pause potential.

with the biological literature is $\epsilon$, which controls the shape of the potential $U_{1}(x)$. The details of the parameter calculations are described below.

A parameter of central importance is the noise intensity parameter $D$ in (1.2), which is also known as the diffusion coefficient, and many other parameters depend on the value of $D$. In order to estimate it, one needs a value for the Boltzmann constant $\left(k_{\beta} T=4.1 \mathrm{pN}\right.$ $\mathrm{nm}[34,35])$ and the friction coefficient, $\gamma$. The latter is given by the sum of the rotational and translational friction. By approximating the enzyme as a sphere of radius $R \approx 6.5 \mathrm{~nm}$ [40], we have

$$
\gamma=6 \pi \eta R+\left(\frac{2 \pi}{10 B P}\right)^{2} 8 \pi \eta R^{3}
$$

as developed in [48], where $\eta$ is the viscosity of the medium, typically water, which is estimated to be $10^{-9} \mathrm{pN} \mathrm{s} / \mathrm{nm}^{2}$, and $B P$ is the distance between nucleotides which is approximately $0.34 \mathrm{~nm}$. Therefore Eq. (7.3) yields $\gamma=2.4 \times 10^{-5} \mathrm{pN} \mathrm{s} / \mathrm{nm}$. Using these values for $k_{\beta} T$ and $\gamma$, we calculate $D \approx 1.7 \times 10^{5} \mathrm{~nm}^{2} / \mathrm{s}$, which agrees well with the experimental value of $1.5 \times 10^{5} \mathrm{~nm}^{2} / \mathrm{s}$ given in [50].

As mentioned above, we are choosing $U_{1}(x)$ to represent the presence of pauses during elongation and $U_{2}(x)$ to represent translocation by one nucleotide. Hence for our purposes $L_{1}=100$ nucleotides, $L_{2}=1$ nucleotide, and we convert $D$ from units measured using nanometers into units involving nucleotides as given below.

$$
D=1.7 \times 10^{5} \mathrm{~nm}^{2} / \mathrm{s} \times \frac{1 \mathrm{nt}^{2}}{0.34^{2} \mathrm{~nm}^{2}}=1.47 \times 10^{6} \mathrm{nt}^{2} / \mathrm{s} .
$$

For ease of computation, an additional rescaling for $D$ is applied, and we compute in terms of $(100 \mathrm{nt})^{2}$, and the value used in model simulations becomes

$$
D=1.47 \times 10^{2}
$$


with units of $(100 \mathrm{nt})^{2} / \mathrm{sec}$. From this, we calculate $F$ based on the desired values for $p_{+}$and $p_{-}$given by Eqs. (4.2) and (4.3). The probabilities are determined from experimental results describing the transcription process. In order to account for backtracking pauses that, while infrequent, do occur during transcription we prescribe $p_{+}\left(L_{2}\right)=0.99$ and $p_{-}\left(L_{2}\right)=0.01$ during the elongation process when the RNAP is not experiencing a pause. This allows the particle to exit the segment $\left[-L_{2}, L_{2}\right]$ occasionally to the left i.e. in the direction opposite that of the elongation. Using Eq. (4.2) and setting $p_{+}=0.99$, and staying consistent with the length scale determined by the rescaling of $D$, one can solve for $F$ in terms of the units of $L_{2}=0.01(100 \mathrm{nt})$,

$$
F=-D \ln \left(\frac{1-0.99}{0.99}\right)
$$

where $D=1.47 \times 10^{2}(100 \mathrm{nt})^{2} / \mathrm{s}$.

We estimate $\epsilon$ somewhat qualitatively by examining the graph of the total effective potential $V(x)=U_{1}(x)+U_{2}(x)-F x$ and the contribution of the pause potential $U_{1}\left(x, L_{1}\right)$ to its behavior. For small values of $\epsilon$ the "base" of the pause potential spreads out and is wider. We prefer to have a very small base so that the graphical behavior of our pause potential resembles that of a Dirac delta function. Fig. 4 displays the graphs of the full potential functions $V(x)$ for four different values of $\epsilon$ (with all other parameters fixed). For the computations presented here, we choose $\epsilon=1000$, but the choice of $\epsilon$ is somewhat arbitrary.

\subsubsection{Estimation of parameters $c_{1}$ and $c_{2}$}

Now we estimate the amplitude of $U_{1}(x)$ and $U_{2}(x), c_{1}$ and $c_{2}$ respectively. These parameters are tuned so that the model is consistent with information from the biological literature. The average velocity of an RNAP transcribing an rrn operon is $90 \mathrm{nt} / \mathrm{s}$ which corresponds to a $60 \mathrm{~s}$ transcription time [39]. Assuming the length of the DNA strand is 5450 nucleotides, as for $E$. Coli, the number of 100 nucleotide segments (space from one pause to another in our model) to compose the strand is 54.5. Therefore, to obtain a 60 second transcription time, the MST from one segment should be 1.1009 seconds. As mentioned previously, pauses range in time duration from 1-5 seconds. To remain biologically consistent and to adhere to the 60 second transcription time, we tune the amplitude of the pause potential, $c_{1}$, to calculate a one second pause. In this case, we use the formula,

$$
\mu_{1}=\frac{1}{D\left(1+e^{\frac{-F L_{1}}{D}}\right)} \int_{0}^{L_{1}} \int_{x-L_{1}}^{x} e^{\frac{U_{1}(x)-F x}{D}} e^{\frac{-\left(U_{1}(y)-F y\right)}{D}} d y d x
$$

and seek to determine $c_{1}$ so that $\mu_{1}=1$ second. After some experimentation, this amplitude is $c_{1}=1790.4138$. Keeping $c_{1}$ fixed, we next consider full multi-periodic potential where the potential contains both the pause and the elongation potentials. The amplitude for this elongation potential, $c_{2}$, is tuned in order to calculate the MST of 1.1009. This amplitude is computed as $c_{2}=20.8067$. The MST is calculated using the original formula in 


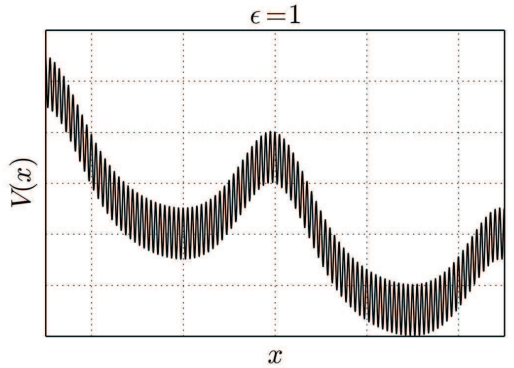

(a) $\epsilon=1$

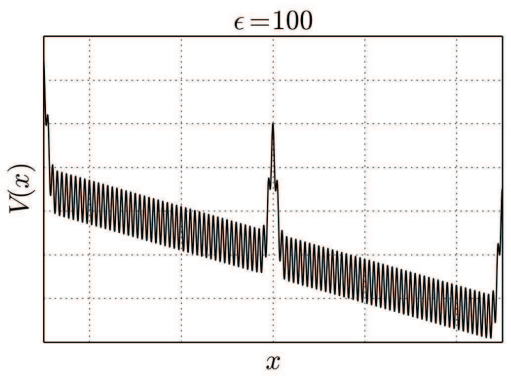

(c) $\epsilon=100$

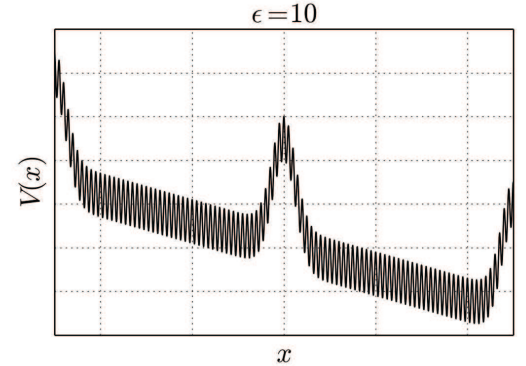

(b) $\epsilon=10$

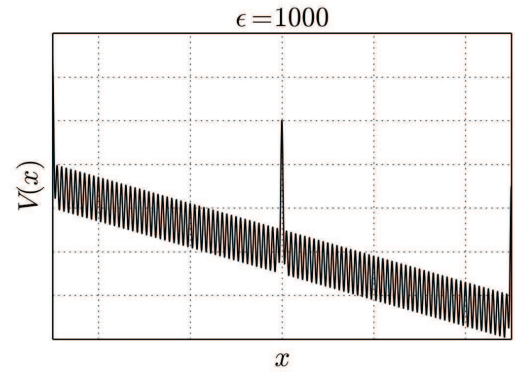

(d) $\epsilon=1000$

Figure 4: The parameter $\epsilon$ affects the shape of the pause potential function. Higher values of $\epsilon$ produce more of a spike, while the lower values of $\epsilon$ create a much less severe and more rounded potential.

Eq. (2.9) and the potential $V(x)=U_{1}\left(x, L_{1}\right)+U_{2}\left(x, L_{2}\right)-F x$ where $U_{1}$ and $U_{2}$ are defined in Eq's (7.1) and (7.2) respectively.

Using the same parameters, we compare the approximation formula, $T^{2}$ with that of the analytical expressions mentioned above. The MST approximation calculated using the formula for $T^{2}$ is 1.01055 seconds. Therefore the difference between the two calculated MSTs is

$$
\left|\mu_{2}-T^{2}\right| \approx 0.090845 \text { seconds }
$$

which is an excellent agreement. The parameters used in these calculations are shown in Table 1.

Table 1: Parameters used in calculations of mean escape time.

\begin{tabular}{||c|c|c|c|c|c|c|c||}
\hline Parameter & $L_{1}$ & $L_{2}$ & $c_{1}$ & $c_{2}$ & $\epsilon$ & $F$ & $D$ \\
\hline Value & 1 & 0.01 & 1790.4138 & 20.8067 & 1000 & 675.5 & $1.47 \times 10^{2}$ \\
\hline
\end{tabular}

We also analyze the impact of the parameter $\epsilon$, by varying $\epsilon$ over a large range of values. These results are shown in Fig. 5. We note that for all values of $\epsilon$ explored here (note the logarithmic scale), the error between the MST and the approximation remains very small. Interestingly, the graph of the mean escape time has a pronounced maximum 


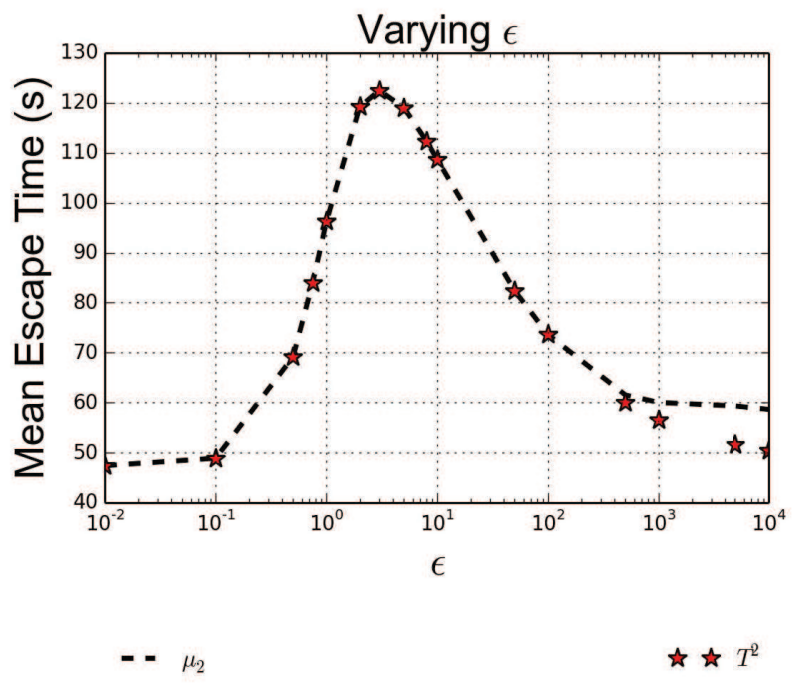

Figure 5: MST for a range of $\epsilon$ values with the calculated MST shown in the black dashed line and the approximation shown in the red stars.

at value $\epsilon \approx 3$ along with points of inflection on either side of this value. At this point, we do not have a theoretical explanation of this effect of the shape of the potential on mean escape time; however, there are results in the literature describing significant enhancement of diffusion effects for values of the tilt parameter that are near a certain threshold, see [44] and [45]. In the current case of the biological application, the tilt parameter is determined according to the predisposition of the RNAP to move in the forward direction along the DNA strand with a certain probability, therefore, the tilt parameter is not free to be tuned further. However, this observation warrants future consideration as to whether a biologically observed phenomena of elongation "speed up" on highly transcribed genes [19] should be investigated in the context of this model. Fig. 5 leads one to conclude that the shape parameter $\epsilon$ can be adjusted so that a certain mean escape time from a transcriptional pause may be achieved.

We conclude this example by noting that our model captures the main features of DNA elongation with the pausing behavior. Using our MST approximation,

$$
T^{2}=2 D v_{2}\left(U_{2}\right) \mu_{1}\left(U_{1}\right),
$$

we only need to recalculate $\mu_{1}\left(U_{1}\right)$ when we change parameters of the pausing process, and $v_{2}\left(U_{2}\right)$ when we change elongation parameters. This efficiency is a clear computational benefit of our approximation formula during the model validation process.

\subsection{Mean escape time using a Fourier expansion for a quadratic wave}

In this example, we use a truncated Fourier expansion in order to approximate a potential function. Mean escape time estimates are computed using the function given by the 
partial sum as well as the multiplicative MST approximation derived in this paper. We examine the errors incurred using each of these approximations. Begin with the quadratic function

$$
U_{0}(x)=-\left(x-\frac{L}{2}\right)^{2}
$$

defined on the interval $[0, L]$. The periodic function is the periodic extension of $U_{0}$ given by

$$
U(x)=-\left((x-n L)-\frac{L}{2}\right)^{2}, \quad x \in[n L,(n+1) L], \quad n \in \mathbb{N} .
$$

We consider the tilted periodic potential

$$
V(x)=U(x)-F x,
$$

where $U(x)$ is the periodic extension given in Eq. (7.7). For this example, we consider $F=0.2$. The graph of this potential on the interval $[0,3 \mathrm{~L}]$ is shown in Fig. 6(a).

Since $U(x)$ is a continuous function and $U^{\prime}(x)$ is piecewise continuous, by the Fourier Convergence Theorem the Fourier series converges uniformly to $U(x)$ for all $x$. The Fourier series for $U(x)$ is given by

$$
U(x)=\frac{-L}{12}-\sum_{n=1}^{\infty}\left(\frac{L}{n \pi}\right)^{2} \cos \left(\frac{2 \pi n x}{L}\right) .
$$

The truncated Fourier series is the finite sum,

$$
U^{N}(x)=\frac{-L}{12}-\sum_{n=1}^{N}\left(\frac{L}{n \pi}\right)^{2} \cos \left(\frac{2 \pi n x}{L}\right) .
$$

The error between the truncated Fourier expansion and the original function decreases with the addition of more terms, and the inclusion of a small number of terms in the sum is required in order to observe good agreement between the original function and its approximation, see Fig. 6(b). To frame the truncated Fourier series in the context of a tilted multi-periodic potential consider the definition

$$
V^{N}(x):=\left(\frac{-L}{12}-\left(\frac{L}{\pi}\right)^{2} \cos \left(\frac{2 \pi x}{L}\right)-F x\right)-\sum_{n=2}^{N}\left(\frac{L}{n \pi}\right)^{2} \cos \left(\frac{2 \pi n x}{L}\right) .
$$

This expression takes the form

$$
V^{N}(x)=\left(U_{1}(x)-F x\right)+\sum_{i=2}^{N} U_{i}(x),
$$

where

$$
U_{i}(x)=-\left(\frac{L}{i \pi}\right)^{2} \cos \left(\frac{2 \pi i x}{L}\right), \quad i=2,3, \cdots, N
$$




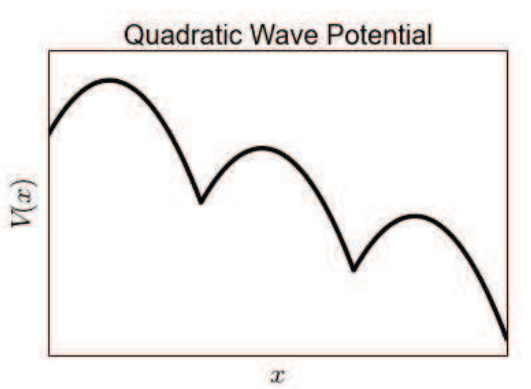

(a) The tilted potential function $V(x)$ with $F=0.2$ and $U(x)$ as the periodic extension given in Eq. (7.7) on the interval $[0,3 L]$.

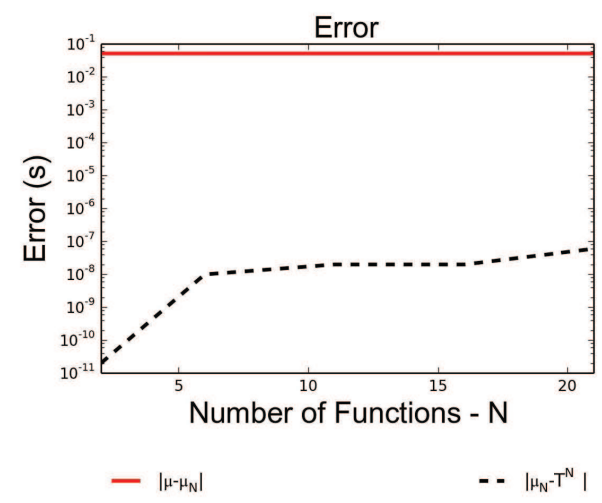

(c) The errors calculated by the differences $\left|\mu-\mu_{N}\right|$ and $\left|\mu_{N}-T^{N}\right|$ are shown in red and black dashes respectively.

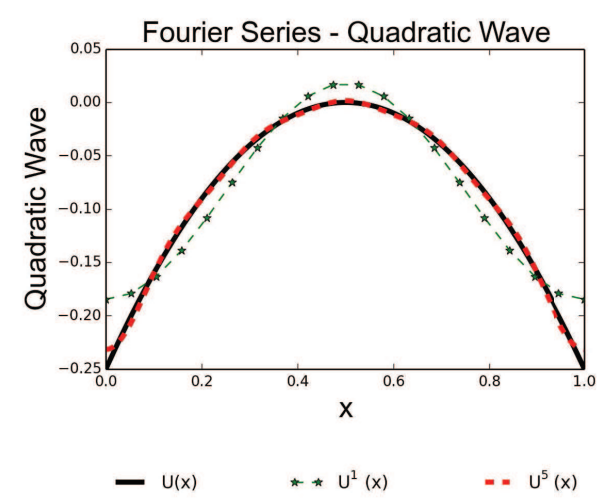

(b) The potential (black) on $[0, L]$ is compared to the Fourier series with one term, $U^{1}(x)$ (green stars) and with five terms, $U^{5}(x)$ (red dashes).

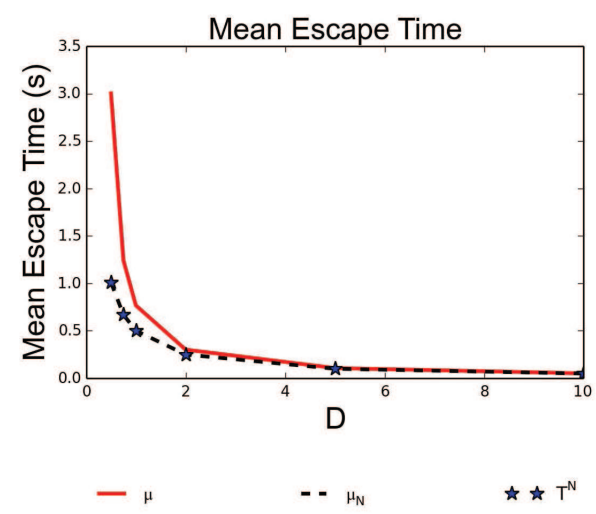

(d) The MST using the quadratic potential $\mu$ and using the Fourier series $\mu_{N}$ are shown in red and black dashes, respectively. The MST approximation, $T^{N}$, using Eq. (3.10), is displayed with blue stars. Results shown for a range of values for $D$ with $N=20$ fixed.

Figure 6: Figures (a) and (b) display the graph of the quadratic potential function $V(x)$ along with some Fourier approximations. Figures (c) and (d) illustrate measures of the error among the various MST calculations for the functions shown in (a) and (b).

has a period of $L_{i}=L / i$ for $i=2,3, \cdots, N$ and

$$
U_{1}(x, L)=\left(\frac{-L}{12}-\left(\frac{L}{\pi}\right)^{2} \cos \left(\frac{2 \pi x}{L}\right)\right) .
$$

The MST for this example is calculated in three ways. The first is to compute the true mean escape time $\mu$ where the function in (7.8) is used in the definition of the MST given 
Table 2: Mean escape times $\mu$ and $\mu_{N}$, with the multiplicative approximation $T^{N}$.

\begin{tabular}{||c|c|c|c|c|c||}
\hline$N$ & $\mu$ & $\mu_{N}$ & $T^{N}$ & $\begin{array}{c}\text { Magnitude of } \\
\left|\mu-\mu_{N}\right|\end{array}$ & $\begin{array}{c}\text { Magnitude of } \\
\left|\mu_{N}-T^{N}\right|\end{array}$ \\
\hline 2 & 0.30184637 & 0.25011249 & 0.25011249 & $10^{-2}$ & $10^{-11}$ \\
6 & 0.30184637 & 0.25013829 & 0.25013828 & $10^{-2}$ & $10^{-8}$ \\
11 & 0.30184637 & 0.25013884 & 0.25013882 & $10^{-2}$ & $10^{-8}$ \\
16 & 0.30184637 & 0.25013890 & 0.25013888 & $10^{-2}$ & $10^{-8}$ \\
21 & 0.30184637 & 0.25013896 & 0.25013890 & $10^{-2}$ & $10^{-8}$ \\
\hline
\end{tabular}

by (2.9). The second is to compute the true mean escape time $\mu_{N}$ for a function which is a finite Fourier approximation $V^{N}$ as given in the Eq. (7.12). The third and final means of approximation considered here is to use the MST labeled $T^{N}$, which is an approximation of $\mu_{N}$. Using the multiplicative approximation from Theorem 3.1, the quantity $T^{N}$ is calculated according to the definition in Eq. (3.10). We examine the sharpness of this estimate by numerically calculating both $\mu_{N}$ and the exact value of $\mu$ using a very accurate quadrature rule for the expression (2.9). For all three calculations we use the parameters $D=2$ and $L=1$. The errors between the MSTs given by the differences $\left|\mu-\mu_{N}\right|$, and $\left|\mu_{N}-T^{N}\right|$ for an increasing number of functions are shown in Fig. 6(c). Also, Table 2 displays the results of $\mu, \mu_{N}$, and $T^{N}$ as well as the differences $\left|\mu-\mu_{N}\right|$ and $\left|\mu_{N}-T^{N}\right|$.

The difference $\left|\mu-\mu_{N}\right|$ is 0.051734 for the truncated function with one term and decreases to 0.051707 for the truncated function with twenty terms. This error given by $\left|\mu-\mu_{N}\right|$ remains very consistent over the addition of terms. As we can see from Fig. 6(b), the Fourier series approximation to $U_{0}(x)$ is already very good at 5 terms. As more terms are added, this error will continue to decrease. From Theorem 6.1, this error will approach zero as the number of terms $N \rightarrow \infty$, but we compare the error in the MST approximation $\left|\mu_{N}-T^{N}\right|$ for small $N$ as that is the context where $T^{N}$ is the most accurate approximation of the mean escape time. This error remains quite small for a reasonably large value of $N$, which determines the number of terms in the finite Fourier approximation.

Next consider the MST for a fixed number of Fourier series terms, $N=20$, while the value of the noise intensity parameter $D$ is allowed to vary. From our discussion of the error estimate we derived Eq. (5.9), and just as in our previous example, as $D$ decreases, the term on the right hand side of Eq. (5.9) increases, and errors are more significant in this approximation. The results of these calculations are displayed in Fig. 6(d). The difference $\left|\mu_{N}-T^{N}\right|$ for $D=0.5$ is on the order of $10^{-5}$. This quantity increases to $10^{-2}$ for $D=0.1$.

While the difference $\left|\mu-\mu_{N}\right|$ does increase dramatically for small values of $D, T^{N}$ remains close to $\mu_{N}$. The difference $\left|\mu-\mu_{N}\right|$ is also expected to increase as $D$ decreases, from the inequality given in the proof of Theorem 6.1. While $\mu_{N} \rightarrow \mu$ as $N \rightarrow \infty$ from 
Theorem 6.1, the difference $\left|\mu-\mu_{N}\right|$ will require more terms of the sequence in order to be made sufficiently small for small values of $D$. As we are holding $N=20$ fixed here, the error will continue to increase as $D$ decreases. For values of $D>1$, Eq. (3.10) provides excellent agreement to Eq. (2.9). This example illustrates that the accuracy of the MST approximation is best used for situations where low numbers of terms in a Fourier series approximation are needed. It also shows that the accuracy of the estimate also depends strongly on the value of $D$, and both of these issues should be given consideration when using this estimate.

\subsection{Potential with two periodic functions}

This section contains a simple example of a potential function consisting of a sum of only two periodic functions, the frequency of the second function being significantly smaller than that of the first. In such a construction, the notation from the previous section implies that $\mu=\mu_{2}$ since the original potential function can be viewed as a finite Fourier expansion. Consider the multi-periodic potential

$$
V(x)=\cos (2 \pi x)+\frac{1}{16} \cos (200 \pi x)-0.6 x .
$$

The first periodic function, $U_{1}(x)=\cos (2 \pi x)$, has period $L_{1}=1$, while the second function, $U_{2}(x)=\frac{1}{16} \cos (200 \pi x)$, is also periodic with least period $L_{2}=1 / 100$. The tilt parameter is set by $F=0.6$. A plot of this potential is shown in Fig. 7(a). The focus of this section is the accuracy of the MST approximation for the situation where it is most suitable, that is, when the largest period of the functions comprising $V(x)$ is considerably larger than that of the smaller period of the other component function of $V(x)$ in (7.15). The error $\left|\mu_{2}-T^{2}\right|$ is examined in detail, and the MST estimate computed using $T^{2}$ is significantly better than the bound given in this paper for some cases. In addition, the role of the noise parameter $D$ is also considered.

The potential $V(x)$ is highly oscillatory but the MST of each individual function is very easy to calculate. The formula for the MST is

$$
\mu_{2}=q \int_{0}^{1} \int_{x-1}^{1} e^{\frac{U_{1}(x)-0.6 x}{D}} e^{\frac{-\left(U_{1}(y)-0.6 y\right.}{D}} e^{\frac{U_{2}(x)}{D}} e^{\frac{-U_{2}(y)}{D}} d y d x
$$

Following the error analysis completed in Lemma 5.1, we have the relationship

$$
\mu_{2}=v_{2} \mu_{1}+q v_{2} \epsilon_{2}=T^{2}+q v_{2} \epsilon_{2} .
$$

We seek to examine the error $\left|\mu_{2}-T^{2}\right|$ as a function of the parameter $D$ using this equation. In order to quantify $\epsilon_{2}$ for this example, we follow the arguments in the proof of Lemma 4.2, and the details of the calculations can be found in Appendix C. Those details include the estimates of $M_{1}$ and $M_{2}$ for this particular example, and using those 


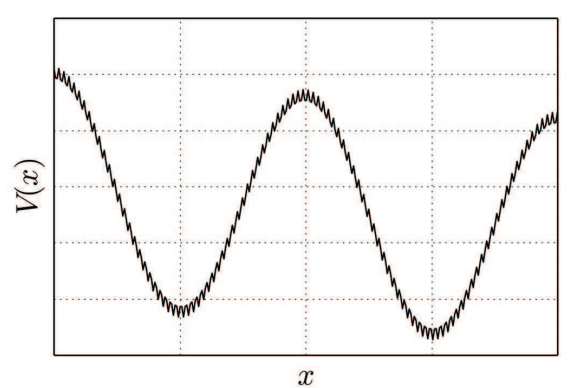

(a) A multi-periodic potential with two periodic functions as given in Eq. (7.15) is shown. It is the sum of two cosine functions defined by periods $L_{1}=1, L_{2}=1 / 100$ and a tilt of $F=0.6$.

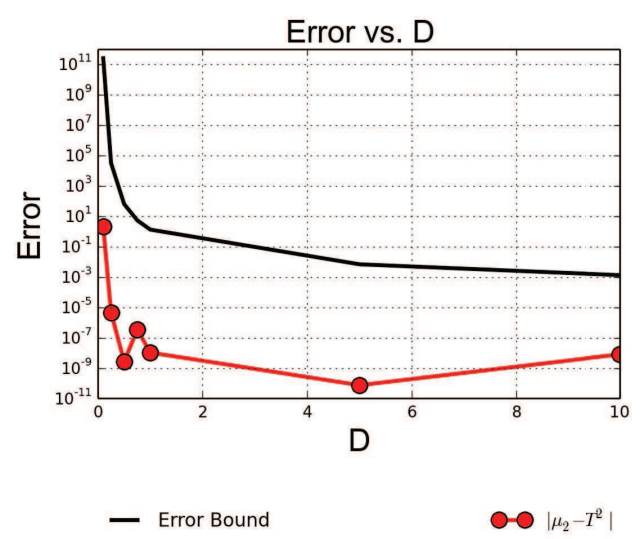

(b) The graph of the error bound is shown in black with the true error in red. The parameter $D$ ranges in the interval $[0.1,10]$. This plot uses a $\log$ scale on the vertical-axis.

Figure 7: Figure (a) displays the graph of the potential function $V(x)$ from Eq. (7.15), and Figure (b) illustrates the behavior of the error of the MST approximation over a range of values for the noise intensity parameter.

estimates, one can express $\epsilon_{2}$ as a function of $D$ where we have the following inequality

$$
\epsilon_{2}(D) \leq \frac{3}{D} M_{1} \kappa^{1 / D} L_{2} \approx \frac{0.2065(10.066)^{1 / D}}{D} .
$$

From Eq. (7.17), we see that the error in the MST approximation is given by $\left|\mu_{2}-T^{2}\right|=$ $q v_{2} \epsilon_{2}$, and that error is bounded by

$$
\left|\mu_{2}-T^{2}\right| \leq q v_{2}\left[\frac{0.2065(10.066)^{1 / D}}{D}\right]=\left(\frac{1}{1+e^{\frac{-0.6}{D}}}\right) v_{2}\left[\frac{0.2065(10.066)^{1 / D}}{D^{2}}\right]
$$

from Eq. (C.3) for the current example.

To examine this error as a function of the noise intensity parameter, $D$, we calculate and report both the true error $\left|\mu_{2}-T^{2}\right|$ along with the value of the error bound given by the right side of Eq. (7.19). These calculations are reported in Table 3 for a variety of different values of $D$. The results depict the very good agreement that we would expect to find with the error decreasing with increasing values of $D$. The error bound and the true error are plotted on the same axes for comparison using a log scale on the $y$-axis, in Fig. 7(b). Although the error bound is valid for the range of $D$, it severely overestimates the true error. In fact, the actual error values $\left|\mu_{2}-T^{2}\right|$ over the entire range of choices for $D$ are quite low indicating that the error using the MST calculation can be much smaller than the prediction of the error bound given in this work.

These three examples detailed in Section 7 demonstrate the utility of the approximation method. With a relatively small error, one can estimate the MST using $T^{N}$ in a 
Table 3: Calculated errors for the MST approximation $T^{2}$ over a range of noise intensity values $D$. The third column contains the value of the error bound given by Eq. (7.19).

\begin{tabular}{||ccc||}
\hline$D$ & $\left|\mu_{2}-T^{2}\right|$ & Error Bound \\
\hline 0.1 & 2.219279971905 & $2.66226934 \times 10^{11}$ \\
0.25 & $4.6258332 \times 10^{-6}$ & $3.208295296 \times 10^{4}$ \\
0.5 & $2.6435893 \times 10^{-9}$ & $6.482483122 \times 10$ \\
0.75 & $3.5115565 \times 10^{-7}$ & 5.5243404968 \\
1 & $1.05732914 \times 10^{-8}$ & 1.3447030926 \\
5 & $7.50403 \times 10^{-11}$ & $6.9475514757 \times 10^{-3}$ \\
10 & $8.1085959 \times 10^{-9}$ & $1.3397311847 \times 10^{-3}$ \\
\hline
\end{tabular}

computationally efficient manner. The ease of computation, especially in light of altering parameters for use in parameter studies, makes the approximation method an asset for research involving repeated calculations of MST.

\section{Discussion}

This paper examines a model of particle transport from one potential well to the next by considering the Fokker-Planck equation over an individual segment of its domain. The particular form of the potential function prescribed in the equation allows us to give a form for the particle's mean escape time (MST) from this segment and hence estimate the dispersion and the drift associated with the overall particle motion. Generally, the MST must be estimated numerically since an algebraic expression of the integral representation is usually intractable, see Eq. (2.9). The focus of our analysis is the estimation of the MST for tilted potential functions of the form $V(x)=U(x)-F x$, where $U(x)$ is periodic with least period $L$. First it is noted that the probability of escaping a segment on the right (or the left) depends on the tilt parameter $F$ and the period $L$, as well as the noise intensity parameter $D$. Consequently, we have shown that when $U(x)$ is constructed using a linear combination of periodic functions where $L$ is a common period of all individual elements within the combination, then the escape probability is unchanged with the addition of more periodic functions into that linear combination, so long as the period of $U(x)$ remains fixed. Hence, the escape probability is independent of the algebraic components of $U(x)$, so long as the least period of the function is given by the parameter $L$. In contrast, the MST for a given potential function $V(x)$ depends explicitly on its algebraic representation, see Eq. (2.9).

The main results of this paper derive an expression that approximates the MST for the case of a tilted periodic potential composed of a sum of several periodic functions. The formula is multiplicative and is given in terms of the MST of each of the individual periodic functions that compose the original potential. This formula, the multiplicative MST 
approximation given by Eq. (3.10) in Theorem 3.1, is valid for a potential that is composed of a linear combination of any finite number of periodic functions. Theorem 3.1 is easily proven with recursion.

A demonstrated application for Eq. (3.10) is its use for repeated calculations during parameter estimation for model validation as shown in Section 7.1. There we were able to determine coefficients for a model of RNAP elongation for DNA transcription that includes transcriptional pausing and achieve model outputs that are consistent with the literature. Another use of Eq. (3.10) is in conjunction with a Fourier series approximation of a potential function, as shown by the quadratic wave example in Section 7.2. When a finite Fourier expansion is used to approximate the potential function, then the multiplicative MST derived here gives an approximation to the true MST of the original potential that is efficient to calculate. In fact, the MST of the individual terms of the Fourier series can be computed a priori and stored for table lookup when needed. An added benefit is that the MST for each individual basis function can be pre-computed to a high degree of accuracy using the formula in Eq. (2.9) and applying an adaptive quadrature rule that can be adjusted to choose a computational grid that is appropriate for the period of the particular function in the Fourier basis. As one adds more terms to the finite approximation of the Fourier series, only the MST of each of the new terms is required to build the new approximation.

Finally, we also examine the effect of the noise intensity parameter on the reliability and accuracy of the MST approximation. For noise intensity $D \geq 1$ there is excellent agreement between the true MST and the multiplicative approximation. We also prove convergence of the MST of the Fourier series to the MST of the limiting function. Quantifying the explicit rate of convergence remains an open problem.

\section{Acknowledgments}

The authors wish to acknowledge support from the National Science Foundation under grant DMS-1226213. Dr. T. Heberling acknowledges support from the Kopriva Graduate Fellowship Program at Montana State University.

\section{A Proof of Theorem 3.2}

Proof. Let the periodic potential be

$$
\begin{aligned}
V^{n}(x) & =U_{1}\left(x, L_{1}\right)+U_{2}\left(x, L_{2}\right)+\cdots+U_{n}\left(x, L_{n}\right)-F x \\
& =\left(U_{1}\left(x, L_{1}\right)-\frac{F x}{n}\right)+\left(U_{2}\left(x, L_{2}\right)-\frac{F x}{n}\right)+\cdots+\left(U_{n}\left(x, L_{n}\right)-\frac{F x}{n}\right)
\end{aligned}
$$

as in Eq. (3.12). Also let $V^{n-1}(x)$ be

$$
V^{n-1}(x)=\left(U_{1}\left(x, L_{1}\right)-\frac{F x}{n}\right)+\left(U_{2}\left(x, L_{2}\right)-\frac{F x}{n}\right)+\cdots+\left(U_{n-1}\left(x, L_{n-1}\right)-\frac{F x}{n}\right)
$$


So

$$
V^{n}(x)=V^{n-1}(x)+\left(U_{n}\left(x, L_{n}\right)-F x / n\right)
$$

Then the MST is

$$
\mu=\frac{1}{D\left(1+e^{\frac{-F L}{D}}\right)} \iint_{\Omega} e^{\frac{V^{n-1}(x)}{D}} e^{\frac{-V^{n-1}(y)}{D}} e^{\frac{U_{n}\left(x, L_{n}\right)-F x / n}{D}} e^{\frac{-\left(U_{n}\left(y, L_{n}\right)-F y / n\right)}{D}} d y d x .
$$

Partitioning the domain into $\Omega_{k, s}$ and using the Weighted Mean Value Theorem for Double Integrals, there exists an $\left(x_{0}(k, s), y_{0}(k, s)\right)$ such that

$$
\begin{aligned}
\mu & =\frac{1}{D\left(1+e^{\frac{-F L}{D}}\right)} \sum_{k=1}^{N_{n}} \sum_{s=1}^{N_{n}} e^{\frac{V^{n-1}\left(x_{0}(k, s)\right)}{D}} e^{\frac{-V^{n-1}\left(y_{0}(k, s)\right)}{D}} \iint_{\Omega_{k, s}} e^{\frac{U_{n}\left(x, L_{n}\right)-F x / n}{D}} e^{\frac{-\left(U_{n}\left(y, L_{n}\right)-F y / n\right)}{D}} d y d x \\
& =\frac{1}{D\left(1+e^{\frac{-F L}{D}}\right)} \sum_{k=1}^{N_{n}} \sum_{s=1}^{N_{n}} e^{\frac{V^{n-1}\left(x_{0}(k, s)\right)}{D}} e^{\frac{-V^{n-1}\left(y_{0}(k, s)\right)}{D}} \iint_{\Omega_{k, s}} e^{\frac{U_{n}\left(x, L_{n}\right)}{D}} e^{\frac{-U_{n}\left(y, L_{n}\right)}{D}} e^{\frac{F(y-x)}{n D}} d y d x .
\end{aligned}
$$

Consider only the integral in Eq. (A.3) and apply the mapping $G_{k, s}: \Omega_{k, s} \rightarrow \Omega_{0, n}$ given by Eq. (4.13),

$$
\begin{aligned}
& \iint_{\Omega_{k, s}} e^{\frac{U_{n}\left(x, L_{n}\right)-F x / n}{D}} e^{\frac{-\left(U_{n}\left(y, L_{n}\right)-F y / n\right)}{D}} d y d x \\
= & \iint_{\Omega_{0, n}} e^{\frac{U_{n}\left(x, L_{n}\right)-F x / n}{D}} e^{\frac{-\left(U_{n}\left(y, L_{n}\right)-F y / n\right)}{D}} e^{\frac{F\left(s-N_{n}\right) L_{n}}{n D}} d y d x \\
= & e^{\frac{F\left(s-N_{n}\right) L_{n}}{n D}} \iint_{\Omega_{0, n}} e^{\frac{U_{n}\left(x, L_{n}\right)-F x / n}{D}} e^{\frac{-\left(U_{n}\left(y, L_{n}\right)-F y / n\right)}{D}} d y d x \\
= & D\left(1+e^{\frac{-F L_{n}}{n D}}\right) \mu\left(U_{n}, L_{n}, F / n\right) e^{\frac{F\left(s-N_{n}\right) L_{n}}{n D}} .
\end{aligned}
$$

With the tilting term included on the function in the integral, an extra term emerges from the integral as a result of the mapping to $\Omega_{0, n}$. Using this for the integral in Eq. (A.3), we have

$$
\mu=\frac{\left(1+e^{\frac{-F L_{n}}{n D}}\right)}{\left(1+e^{\frac{-F L}{D}}\right)} \mu\left(U_{n}, L_{n}, \frac{F}{n}\right) \sum_{k=1}^{N_{n}} \sum_{s=1}^{N_{n}} e^{\frac{V^{n-1}\left(x_{0}(k, s)\right)}{D}} e^{\frac{-V^{n-1}\left(y_{0}(k, s)\right)}{D}} e^{\frac{F\left(s-N_{n}\right) L_{n}}{n D}} .
$$

Note,

$$
e^{\frac{F\left(1-N_{n}\right) L_{n}}{n D}} \leq e^{\frac{F\left(s-N_{n}\right) L_{n}}{n D}} \leq e^{\frac{F\left(N_{n}-N_{n}\right) L_{n}}{n D}}=1
$$

Therefore

$$
\begin{aligned}
& \frac{\left(1+e^{\frac{-F L_{n}}{n D}}\right)}{\left(1+e^{\frac{-F L}{D}}\right)} \mu\left(U_{n}, L_{n}, \frac{F}{n}\right) e^{\frac{F\left(1-N_{n}\right) L_{n}}{n D}} \sum_{k=1}^{N_{n}} \sum_{s=1}^{N_{n}} e^{\frac{V^{n-1}\left(x_{0}(k, s)\right)}{D}} e^{\frac{-V^{n-1}\left(y_{0}(k, s)\right)}{D}} \\
\leq & \mu \leq \frac{\left(1+e^{\frac{-F L_{n}}{n D}}\right)}{\left(1+e^{\frac{-F L}{D}}\right)} \mu\left(U_{n}, L_{n}, \frac{F}{n}\right) \sum_{k=1}^{N_{n}} \sum_{s=1}^{N_{n}} e^{\frac{V^{n-1}\left(x_{0}(k, s)\right)}{D}} e^{\frac{-V^{n-1}\left(y_{0}(k, s)\right)}{D}} .
\end{aligned}
$$


Consider the term $\mu\left(U_{n}, L_{n}, \frac{F}{n}\right)$. We would like to determine what happens when we change the period. Begin with the integral for $\mu\left(U_{n}, L_{n}, F / n\right)$,

$$
\mu\left(U_{n}, L_{n}, F / n\right)=\frac{1}{D\left(1+e^{\frac{-F L_{n}}{n D}}\right)} \int_{0}^{L_{n}} \int_{x-L_{n}}^{x} e^{\frac{U_{n}\left(x, L_{n}\right)-F x / n}{D}} e^{\frac{-\left(U_{n}\left(y, L_{n}\right)-F y / n\right)}{D}} d y d x .
$$

Making the substitution

$$
z(x)=\frac{1}{L_{n}} x, \quad d z(x)=\frac{1}{L_{n}} d x
$$

we have

$$
\begin{aligned}
\mu\left(U_{n}, L_{n}, F / n\right) & =\frac{1}{D\left(1+e^{\frac{-F L_{n}}{n D}}\right)} \int_{0}^{1} \int_{z(x)-1}^{z(x)} \frac{e^{\frac{\widehat{U}_{n}(z(x), 1)-F L_{n} z(x) / n}{D}}}{e^{\frac{\hat{U}_{n}(z(y), 1)-F L_{n} z(y) / n}{D}}}\left(L_{n}\right)^{2} d z(y) d z(x) \\
& =\left(L_{n}\right)^{2} \mu\left(\widehat{U}_{n}, 1, F L_{n} / n\right),
\end{aligned}
$$

where $\widehat{U}_{n}(z(x), 1)=U_{n}\left(x, L_{n}\right)$. Therefore

$$
\left(L_{n}\right)^{2} \mu\left(\widehat{U}_{n}, 1, \frac{F L_{n}}{n}\right)=\mu\left(U_{n}, L_{n}, F / n\right) .
$$

Returning to Eq. (A.6), we compute

$$
\begin{aligned}
& \frac{\left(1+e^{\frac{-F L_{n}}{n D}}\right)}{\left(1+e^{\frac{-F L}{D}}\right)} \mu\left(\widehat{U}_{n}, 1, \frac{F}{n} L_{n}\right) e^{\frac{F\left(1-N_{n}\right) L_{n}}{n D}} \sum_{k=1}^{N_{n}} \sum_{s=1}^{N_{n}} e^{\frac{V^{n-1}\left(x_{0}(k, s)\right)}{D}} e^{\frac{-V^{n-1}\left(y_{0}(k, s)\right)}{D}}\left(L_{n}\right)^{2} \\
\leq & \mu \leq \frac{\left(1+e^{\frac{-F L_{n}}{n D}}\right)}{\left(1+e^{\frac{-F L}{D}}\right.} \mu\left(\widehat{U}_{n}, 1, \frac{F}{n} L_{n}\right) \sum_{k=1}^{N_{n}} \sum_{s=1}^{N_{n}} e^{\frac{V^{n-1}\left(x_{0}(k, s)\right)}{D}} e^{\frac{-V^{n-1}\left(y_{0}(k, s)\right)}{D}}\left(L_{n}\right)^{2} .
\end{aligned}
$$

Let $\eta_{m}=D\left(1+e^{\frac{-F L_{m}}{n D}}\right) \mu\left(\widehat{U}_{m}, 1, \frac{F}{n} L_{m}\right)$. We can simplify Eq. (A.9) as

$$
\begin{aligned}
& \frac{\eta_{n}}{D\left(1+e^{\frac{-F L}{D}}\right)} e^{\frac{F\left(1-N_{n}\right) L_{n}}{n D}} \sum_{k=1}^{N_{n}} \sum_{s=1}^{N_{n}} e^{\frac{V^{n-1}\left(x_{0}(k, s)\right)}{D}} e^{\frac{-V^{n-1}\left(y_{0}(k, s)\right)}{D}}\left(L_{n}\right)^{2} \\
\leq & \mu \leq \frac{\eta_{n}}{D\left(1+e^{\frac{-F L}{D}}\right)} \sum_{k=1}^{N_{n}} \sum_{s=1}^{N_{n}} e^{\frac{V^{n-1}\left(x_{0}(k, s)\right)}{D}} e^{\frac{-V^{n-1}\left(y_{0}(k, s)\right)}{D}}\left(L_{n}\right)^{2} .
\end{aligned}
$$

Using Lemma 4.2, we have

$$
\begin{aligned}
& \frac{\eta_{n}}{D\left(1+e^{\frac{-F L}{D}}\right)} e^{\frac{F\left(1-N_{n}\right) L_{n}}{n D}} \iint_{\Omega} e^{\frac{V^{n-1}(x)}{D}} e^{\frac{-V^{n-1}(y)}{D}} d y d x+\mathcal{O}\left(L_{n}\right) \\
\leq & \mu \leq \frac{\eta_{n}}{D\left(1+e^{\frac{-F L}{D}}\right)} \iint_{\Omega} e^{\frac{V^{n-1}(x)}{D}} e^{\frac{-V^{n-1}(y)}{D}} d y d x+\mathcal{O}\left(L_{n}\right) .
\end{aligned}
$$


Now we repeat this process. Consider just the integral

$$
\begin{aligned}
& \iint_{\Omega} e^{\frac{V^{n-1}(x)}{D}} e^{\frac{-V^{n-1}(y)}{D}} d y d x \\
& =\iint_{\Omega} e^{\frac{V^{n-2}(x)}{D}} e^{\frac{-V^{n-2}(y)}{D}} e^{\frac{U_{n-1}\left(x, L_{n-1}\right)-F x / n}{D}} e^{\frac{-\left(U_{n-1}\left(y, L_{n-1}\right)-F y / n\right)}{D}} d y d x \\
& =\sum_{k=1}^{N_{n-1}} \sum_{s=1}^{N_{n-1}} e^{\frac{V^{n-2}\left(x_{0}(k, s)\right)}{D}} e^{\frac{-V^{n-2}\left(y_{0}(k, s)\right)}{D}} \\
& \times \iint_{\Omega_{k, s}} e^{\frac{U_{n-1}\left(x, L_{n-1}\right)-F x / n}{D}} e^{\frac{-\left(U_{n-1}\left(y, L_{n-1}\right)-F y / n\right)}{D}} d y d x \\
& =\sum_{k=1}^{N_{n-1}} \sum_{s=1}^{N_{n-1}} e^{\frac{V^{n-2}\left(x_{0}(k, s)\right)}{D}} e^{\frac{-V^{n-2}\left(y_{0}(k, s)\right)}{D}} \\
& \times \iint_{\Omega_{0}} e^{\frac{U_{n-1}\left(x, L_{n-1}\right)-F x / n}{D}} e^{\frac{-\left(U_{n-1}\left(y, L_{n-1}\right)-F y / n\right)}{D}} e^{\frac{F\left(s-N_{n-1}\right) L_{n-1}}{n D}} d y d x \\
& =D\left(1+e^{\frac{-F L_{n-1}}{n D}}\right) \mu\left(U_{n-1}, L_{n-1}, \frac{F}{n}\right) \sum_{k=1}^{N_{n-1}} \sum_{s=1}^{N_{n-1}} e^{\frac{V^{n-2}\left(x_{0}(k, s)\right)}{D}} e^{\frac{-V^{n-2}\left(y_{0}(k, s)\right)}{D}} e^{\frac{F\left(s-N_{n-1}\right) L_{n-1}}{n D}} .
\end{aligned}
$$

Then we have the inequality

$$
e^{\frac{F\left(1-N_{n-1}\right) L_{n-1}}{n D}} \leq e^{\frac{F\left(s-N_{n-1}\right) L_{n-1}}{n D}} \leq e^{\frac{F\left(N_{n-1}-N_{n-1}\right) L_{n-1}}{n D}}=1 .
$$

Using this inequality in Eq. (A.12), we calculate

$$
\begin{aligned}
\frac{\eta_{n} D\left(1+e^{\frac{-F L_{n-1}}{n D}}\right)}{D\left(1+e^{\frac{-F L}{D}}\right)} \mu\left(U_{n-1}, L_{n-1}, \frac{F}{n}\right) e^{\frac{F\left(1-N_{n}\right) L_{n}}{n D}} e^{\frac{F\left(1-N_{n-1}\right) L_{n-1}}{n D}} \\
\quad \times \sum_{k=1}^{N_{n-1}} \sum_{s=1}^{N_{n-1}} e^{\frac{V^{n-2}\left(x_{0}\right)}{D}} e^{\frac{-V^{n-2}\left(y_{0}\right)}{D}}+\mathcal{O}\left(L_{n}\right) \\
\leq \mu \leq \frac{\eta_{n} D\left(1+e^{\frac{-F L_{n-1}}{n D}}\right) \mu\left(U_{n-1}, L_{n-1}, \frac{F}{n}\right)}{D\left(1+e^{\frac{-F L}{D}}\right)} \\
\quad \times \sum_{k=1}^{N_{n-1} \sum_{s=1}^{N_{n-1}} e^{\frac{V^{n-2}\left(x_{0}\right)}{D}} e^{\frac{-V^{n-2}\left(y_{0}\right)}{D}}+\mathcal{O}\left(L_{n}\right) .}
\end{aligned}
$$

This simplifies to

$$
\begin{aligned}
& \frac{\eta_{n} \cdot \eta_{n-1}}{D\left(1+e^{\frac{-F L}{D}}\right)} e^{\frac{F\left(1-N_{n}\right) L_{n}}{n D}} e^{\frac{F\left(1-N_{n-1}\right) L_{n}}{n D}} \sum_{k=1}^{N_{n-1}} \sum_{s=1}^{N_{n-1}} e^{\frac{V^{n-2}\left(x_{0}\right)}{D}} e^{\frac{-V^{n-2}\left(y_{0}\right)}{D}}\left(L_{n-1}\right)^{2}+\mathcal{O}\left(L_{n}\right) \\
\leq & \mu \leq \frac{\eta_{n} \cdot \eta_{n-1}}{D\left(1+e^{\frac{-F L}{D}}\right)} \sum_{k=1}^{N_{n-1}} \sum_{s=1}^{N_{n-1}} e^{\frac{V^{n-2}\left(x_{0}\right)}{D}} e^{\frac{-V^{n-2}\left(y_{0}\right)}{D}}\left(L_{n-1}\right)^{2}+\mathcal{O}\left(L_{n}\right) .
\end{aligned}
$$


Using Lemma 4.2, we compute

$$
\begin{aligned}
& \frac{\eta_{n} \cdot \eta_{n-1}}{D\left(1+e^{\frac{-F L}{D}}\right)} e^{\frac{F\left(1-N_{n}\right) L_{n}}{n D}} e^{\frac{F\left(1-N_{n-1}\right) L_{n-1}}{n D}} \iint_{\Omega} e^{\frac{V^{n-2}(x)}{D}} e^{\frac{-V^{n-2}(y)}{D}} d y d x+\mathcal{O}\left(L_{n-1}\right) \\
\leq & \mu \leq \frac{\eta_{n} \cdot \eta_{n-1}}{D\left(1+e^{\frac{-F L}{D}}\right)} \iint_{\Omega} e^{\frac{V^{n-2}(x)}{D}} e^{\frac{-V^{n-2}(y)}{D}} d y d x+\mathcal{O}\left(L_{n-1}\right) .
\end{aligned}
$$

If we continue this algorithm for all $n$ functions, we will eventually have the formula

$$
\begin{aligned}
& \frac{\prod_{i=2}^{n} \eta_{i}}{D\left(1+e^{\frac{-F L}{D}}\right)} \prod_{i=2}^{n} e^{\frac{F\left(1-N_{i}\right) L_{i}}{n D}} \iint_{\Omega} e^{\frac{U_{1}\left(x, L_{1}\right)-F x / n}{D}} e^{\frac{-\left(U_{1}\left(y, L_{1}\right)-F y / n\right)}{D}} d y d x+\mathcal{O}\left(L_{2}\right) \\
\leq & \mu \leq \frac{\prod_{i=2}^{n} \eta_{i}}{D\left(1+e^{\frac{-F L}{D}}\right)} \iint_{\Omega} e^{\frac{U_{1}\left(x, L_{1}\right)-F x / n}{D}} e^{\frac{-\left(U_{1}\left(y, L_{1}\right)-F y / n\right)}{D}} d y d x+\mathcal{O}\left(L_{2}\right) .
\end{aligned}
$$

This leads to our final formula

$$
\begin{aligned}
& \frac{\left(1+e^{\frac{-F L}{n D}}\right)}{\left(1+e^{\frac{-F L}{D}}\right)}\left(\prod_{i=2}^{n} e^{\frac{F\left(1-N_{i}\right) L_{i}}{n D}} \eta_{i}\right) \mu\left(U_{1}, L, F / n\right)+\mathcal{O}\left(L_{2}\right) \\
\leq & \mu \leq \frac{\left(1+e^{\frac{-F L}{n D}}\right)}{\left(1+e^{\frac{-F L}{D}}\right)}\left(\prod_{i=2}^{n} \eta_{i}\right) \mu\left(U_{1}, L, F / n\right)+\mathcal{O}\left(L_{2}\right) .
\end{aligned}
$$

The proof is completed.

\section{B Proof of Theorem 4.2}

Proof. Since $f$ is continuous on a closed and bounded $\Omega$ there exists a minimum and maximum of $f$ on this domain, $m=\min _{\Omega}(f(x, y))$ and $M=\max _{\Omega}(f(x, y))$. Because $g$ is a positive function, we have

$$
m g(x, y) \leq f(x, y) g(x, y) \leq M f(x, y)
$$

Therefore

$$
m \iint_{\Omega} g(x, y) d y d x \leq \iint_{\Omega} f(x, y) g(x, y) d y d x \leq M \iint_{\Omega} g(x, y) d y d x .
$$

Also, since $g$ is positive

$$
\iint_{\Omega} g(x, y) d y d x>0
$$


Hence

$$
m \leq \frac{\iint_{\Omega} f(x, y) g(x, y) d y d x}{\iint_{\Omega} g(x, y) d y d x} \leq M
$$

and by the Intermediate Value Theorem, there exists a point $\left(s_{0}, t_{0}\right) \in \Omega$ such that

$$
f\left(s_{0}, t_{0}\right)=\frac{\iint_{\Omega} f(x, y) g(x, y) d y d x}{\iint_{\Omega} g(x, y) d y d x}
$$

Therefore

$$
f\left(s_{0}, t_{0}\right) \iint_{\Omega} g(x, y) d y d x=\iint_{\Omega} f(x, y) g(x, y) d y d x
$$

The proof is completed.

\section{Calculations for Example 7.3}

In order to quantify $\epsilon_{2}$ for this example, we follow the arguments in the proof of Lemma 4.2 and examine the expression for $\epsilon_{k, s}$ where

$$
\epsilon_{k, S}=\left|\iint_{\Omega_{k, S}} e^{\frac{U_{1}(x)-F x}{D}} e^{\frac{-\left(U_{1}(y)-F y\right)}{D}} d y d x-e^{\frac{U_{1}\left(x^{\star}\right)-F x^{\star}}{D}} e^{\frac{-\left(U_{1}\left(y^{\star}\right)-F y^{\star}\right)}{D}} L_{2}^{2}\right| .
$$

This leads to an equation similar to (4.21), given by the following for this example

$$
\epsilon_{k, s} \leq \frac{1}{D} \max _{z \in[-L, L]}\left|U_{1}^{\prime}(z)-F\right| \max _{\left(z_{1}, z_{2}\right) \in \Omega}\left|\frac{e^{\frac{U_{1}\left(z_{1}\right)-F z_{1}}{D}}}{e^{\frac{U_{1}\left(z_{2}\right)-F z_{2}}{D}}}\right|\left(\iint_{\Omega_{k, s}}\left|x-x^{\star}\right|+\left|y-y^{\star}\right| d y d x\right) .
$$

Summing the error over all $100^{2}$ parallelograms $\Omega_{k, s}$, one obtains the expression derived in Eq. (5.7) which for this case is

$$
\epsilon_{2} \leq \frac{1}{D} M_{1} M_{2} 3 L_{2}^{3}(100)^{2}=\frac{3}{D} M_{1} M_{2} L_{2}
$$

where

$$
M_{1}=\max _{z \in[-L, L]}\left|U_{1}^{\prime}(z)-F\right|, \quad M_{2}=\max _{\left(z_{1}, z_{2}\right) \in \Omega}\left|\frac{e^{\frac{U_{1}\left(z_{1}\right)-F z_{1}}{D}}}{e^{\frac{U_{1}\left(z_{2}\right)-F z_{2}}{D}}}\right| .
$$

For the calculation of $M_{1}$, we apply principles of Calculus by finding the critical values. The function to be optimized is

$$
U_{1}^{\prime}(z)-F=-2 \pi \sin (2 \pi z)-F
$$

and its derivative is

$$
U_{1}^{\prime \prime}(z)=-4 \pi^{2} \cos (2 \pi z)
$$


The critical values are described by $z=\frac{2 n-1}{4}$ for $n$ any integer. Since we only consider $z \in[-L, L]=[-1,1]$, we have $z= \pm 3 / 4, \pm 1 / 4$ as the admissible critical values. Plugging into the original equation, we have the critical values of $3 / 4$ and $-1 / 4$ which leads to a maximum value of $|-2 \pi-F|$. Since $F=0.6$ in this example, we have

$$
M_{1} \approx 6.883 \text {. }
$$

In order to calculate $M_{2}$, we must solve a multivariable optimization problem. Recall that

$$
M_{2}=\max _{\left(z_{1}, z_{2}\right) \in \Omega}\left|\frac{e^{\frac{U_{1}\left(z_{1}\right)-F z_{1}}{D}}}{e^{\frac{U_{1}\left(z_{2}\right)-F z_{2}}{D}}}\right|=\max _{\left(z_{1}, z_{2}\right) \in \Omega}\left|e^{\cos \left(2 \pi z_{1}\right)-\cos \left(2 \pi z_{2}\right)+F\left(z_{2}-z_{1}\right)}\right|^{1 / D}=\kappa^{1 / D} .
$$

We calculate

$$
\kappa=\max _{\left(z_{1}, z_{2}\right) \in \Omega}\left|e^{\cos \left(2 \pi z_{1}\right)-\cos \left(2 \pi z_{2}\right)+F\left(z_{2}-z_{1}\right)}\right|
$$

by first defining

$$
g\left(z_{1}, z_{2}\right)=e^{\cos \left(2 \pi z_{1}\right)-\cos \left(2 \pi z_{2}\right)+F\left(z_{2}-z_{1}\right)}
$$

and then finding the critical points. The partial derivatives of $g\left(z_{1}, z_{2}\right)$ are

$$
\frac{\partial g}{\partial z_{1}}=\left(-2 \pi \sin \left(2 \pi z_{1}\right)-F\right) e^{\cos \left(2 \pi z_{1}\right)-\cos \left(2 \pi z_{2}\right)+F\left(z_{2}-z_{1}\right)}
$$

and

$$
\frac{\partial g}{\partial z_{2}}=\left(2 \pi \sin \left(2 \pi z_{2}\right)+F\right) e^{\cos \left(2 \pi z_{1}\right)-\cos \left(2 \pi z_{2}\right)+F\left(z_{2}-z_{1}\right)},
$$

and these expressions are both equal to zero when

$$
z_{i}^{*}=\frac{1}{2 \pi} \sin ^{-1}\left(\frac{-F}{2 \pi}\right)+n / 2, \quad i=1,2, \quad n=1,2 .
$$

For the value of $F=0.6$, we have

$$
z_{i}^{*} \approx-0.485,-0.015,0.515,0.985, \quad z_{i} \in \Omega .
$$

Upon inspection, the largest value that $g\left(z_{1}, z_{2}\right)$ attains over this set of critical values is approximately 10.066. One must also check for maximizers of $|g|$ along the boundary of the parallelogram, but in this particular example, the maximum does not occur on the boundary. After examining all the critical points and the points along the boundary, we have determined that

$$
\mathcal{\kappa} \approx \max _{\left(z_{1}, z_{2}\right) \in \Omega}\left|e^{\cos \left(2 \pi z_{1}\right)-\cos \left(2 \pi z_{2}\right)+F\left(z_{2}-z_{1}\right)}\right|=10.066 .
$$

Therefore

$$
M_{2} \approx \kappa^{1 / D}=10.066^{1 / D} .
$$




\section{References}

[1] E. A. Abbondanzieri, W. J. Greenleaf, J. W. Shaevitz, R. Landick, and S. M. Block. Direct observation of base-pair stepping by RNA polymerase. Nature, 438(7067):460-465, 2005.

[2] T. Apostol. Calculus. One-Variable Calculus with an Introduction to Linear Algebra, volume 1. John Wilay and Sons, 1966.

[3] L. Bai, A. Shundrovsky, and M. D. Wang. Sequence-dependent kinetic model for transcription elongation by RNA polymerase. Journal of Molecular Biology, 344(2):335-349, 2004.

[4] G. Bar-Nahum, V. Epshtein, A. E. Ruckenstein, R. Rafikov, A. Mustaev, and E. Nudler. A ratchet mechanism of transcription elongation and its control. Cell, 120(2):183-193, 2005.

[5] M. Bier. Brownian ratchets in physics and biology. Contemporary Physics, 38(6):371-379, 1997.

[6] P. C. Bressloff and J. M. Newby. Stochastic models of intracellular transport. Reviews of Modern Physics, 85(1):135, 2013.

[7] R. V. Churchill. Fourier Series and Boundary Value Problems, volume 2. McGraw-Hill, Inc., 1963.

[8] W. Coffey, Y. P. Kalmykov, S. Titov, and B. Mulligan. Thermally activated escape rate for a Brownian particle in a tilted periodic potential for all values of the dissipation. Physical Review E, 73(6):061101, 2006.

[9] W. Coffey, Y. P. Kalmykov, S. Titov, and B. Mulligan. Semiclassical master equation in wigners phase space applied to brownian motion in a periodic potential. Physical Review E, 75(4):041117, 2007.

[10] W. T. Coffey, M. W. Evans, and P. Grigolini. Molecular diffusion and spectra. WileyInterscience, 1984.

[11] W. T. Coffey and Y. P. Kalmykov. The Langevin equation: with applications to stochastic problems in physics, chemistry and electrical engineering. World Scientific.

[12] W. T. Coffey, Y. P. Kalmykov, and E. Massawe. Effective-eigenvalue approach to the nonlinear langevin equation for the brownian motion in a tilted periodic potential: Application to the josephson tunneling junction. Physical Review E, 48(1):77, 1993.

[13] W. T. Coffey, Y. P. Kalmykov, and E. Massawe. Effective-eigenvalue approach to the nonlinear langevin equation for the brownian motion in a tilted periodic potential. II. application to the ring-laser gyroscope. Physical Review E, 48(2):699, 1993.

[14] D. R. Cox. Renewal Theory. Methuen and Co LTD, 1962.

[15] M. Dangkulwanich, T. Ishibashi, L. Bintu, and C. Bustamante. Molecular mechanisms of transcription through single-molecule experiments. Chemical Reviews, 114(6):3203-3223, 2014.

[16] J. Davenport, J. Wuite, R. Landick, and C. Bustamante. Single-molecule study of transcriptional pausing and arrest by E. coli RNA polymerase. Science, 287(5462):2497-2500, 2000.

[17] M. Depken, E. Galburt, and S. Grill. The origin of short transcriptional pauses. Biophysical Journal, 96(6):2189-2193, 2009.

[18] A. Einstein. Über die von der molekularkinetischen theorie der wärme geforderte bewegung von in ruhenden flüssigkeiten suspendierten teilchen. Annalen der physik, 4, 1905.

[19] V. Epshtein and E. Nudler. Cooperation between RNA polymerase molecules in transcription elongation. Science, 300(5620):801-805, 2003.

[20] R. Ferrando, R. Spadacini, and G. Tommei. Exact solution of the Kramers problem in periodic potentials. Physical Review A, 46(2):R699, 1992.

[21] R. Ferrando, R. Spadacini, and G. Tommei. Kramers problem in periodic potentials: Jump rate and jump lengths. Physical Review E, 48(4):2437, 1993. 
[22] R. Ferrando, R. Spadacini, G. Tommei, and V. Mel'nikov. Numerical test of finite-barrier corrections for the hopping rate in the underdamped regime. Physical Review E, 51(3):R1645, 1995.

[23] R. P. Feynman, R. B. Leighton, and M. Sands. The Feynman Lectures on Physics, Desktop Edition Volume I, volume 1. Basic Books, 2013.

[24] C. W. Gardiner. Handbook of Stochastic Methods, volume 3. Springer, 2004.

[25] C. Giardina. Bounds on the truncation error of periodic signals. Circuit Theory, IEEE Transactions On, 19(2):206-207,1972.

[26] R. Guajardo and R. Sousa. A model for the mechanism of polymerase translocation. Journal of Molecular Biology, 265(1):8-19, 1997.

[27] Q. Guo and R. Sousa. Translocation by T7 RNA polymerase: a sensitively poised Brownian ratchet. Journal of Molecular Biology, 358(1):241-254, 2006.

[28] G. Kassavetis and M. Chamberlin. Pausing and termination of transcription within the early region of bacteriophage T7 DNA in vitro.

[29] R. Kingston and M. Chamberlin. Pausing and attenuation of in vitro transcription in the rrnB operon of E. Coli, R. E. Kingston, M. J. Chamberlin. Cell, 27(3 Pt 2):523-531, 1981.

[30] T. W. Körner. Fourier Analysis. Cambridge university press, 1989.

[31] H. A. Kramers. Brownian motion in a field of force and the diffusion model of chemical reactions. Physica, 7(4):284-304, 1940.

[32] M. H. Larson, R. Landick, and S. M. Block. Single-molecule studies of RNA polymerase: One singular sensation, every little step it takes. Molecular Cell, 41(3):249-262, 2011.

[33] B. Lindner, M. Kostur, and L. Schimansky-Geier. Optimal diffusive transport in a tilted periodic potential. Fluctuation and Noise Letters, 1(1), 2001.

[34] J. Ma, L. Bai, and M. Wang. Transcription under torsion: Supplemental materials. Science, 340(1580), 2013.

[35] J. Marko and E. Siggia. Stretching DNA. Macromolecules, 28:8759-8770, 1995.

[36] B. Matkowsky, Z. Schuss, and E. Ben-Jacob. A singular perturbation approach to Kramers' diffusion problem. SIAM Journal on Applied Mathematics, 42(4):835-849, 1982.

[37] V. Mel'nikov and S. Meshkov. Theory of activated rate processes: Exact solution of the Kramers problem. The Journal of chemical physics, 85(2):1018-1027, 1986.

[38] V. I. Mel'nikov. The Kramers problem: Fifty years of development. Physics Reports, 209(12):1-71, 1991.

[39] K. C. Neuman, E. A. Abbondanzieri, R. Landick, J. Gelles, and S. M. Block. Ubiquitous transcriptional pausing is independent of RNA polymerase backtracking. Cell, 115(4):437447, 2003.

[40] N. Opalka, J. Brown, W. Lane, K. Twist, and R. Landick. Complete structural model of Escherichia coli RNA polymerase from a hybrid approach. PLOS Biology, 2010.

[41] H. Qian. A stochastic analysis of a Brownian ratchet model for actin-based motility and integrate-and-firing neurons. Molecular and Cellular Biomechanics, 1:267-278, 2004.

[42] P. Reimann. Brownian motors: noisy transport far from equilibrium. Physics Reports, 361(2):57-265, 2002.

[43] P. Reimann, C. Van den Broeck, H. Linke, P. Hänggi, J. Rubi, and A. Pérez-Madrid. Diffusion in tilted periodic potentials: Enhancement, universality, and scaling. Physical Review E, 65(3):031104, 2002.

[44] P. Reimann, C. Van den Broeck, H. Linke, P. Hänggi, J. M. Rubi, and A. Pérez-Madrid. Giant acceleration of free diffusion by use of tilted periodic potentials. Phys. Rev. Lett., 87:010602, Jun 2001. 
[45] P. Reimann, C. Van den Broeck, H. Linke, P. Hänggi, J. M. Rubi, and A. Pérez-Madrid. Diffusion in tilted periodic potentials: Enhancement, universality, and scaling. Phys. Rev. E, 65:031104, Feb 2002.

[46] H. Risken. Fokker-Planck Equation. Springer, 1984.

[47] W. Rudin. Principles of Mathematical Analysis, volume 3. McGraw-Hill New York, 1964.

[48] J. Schurr. The one-dimensional diffusion coefficient of proteins absorbed on DNA. Hydrodynamic considerations. Biophysical Chemistry, 9:413-414, 1979.

[49] D.-A. Silva, D. R. Weiss, F. P. Avila, L.-T. Da, M. Levitt, D. Wang, and X. Huang. Millisecond dynamics of RNA polymerase II translocation at atomic resolution. Proceedings of the National Academy of Sciences, 111(21):7665-7670, 2014.

[50] P. Singer and C. Wu. Promoter search by Escherichia coli RNA polymerase on a circular DNA template. Journal of Biological Chemistry, 262(29):14178-14189, 1987.

[51] M. Smoluchowski. Experimentell nachweisbare, der üblichen thermodynamik widersprechende molekularphänomene. Pisma Mariana Smoluchowskiego, 1(2):226-251, 1927.

[52] L. Squire, D. Berg, F. E. Bloom, S. Du Lac, A. Ghosh, and N. C. Spitzer. Fundamental Neuroscience. Academic Press, 2012. 